\title{
ON THE TOPOLOGICAL BOUNDARY OF THE RANGE OF SUPER-BROWNIAN MOTION
}

\author{
By Jieliang Hong ${ }^{1, *}$, LeOnid Mytnik $^{2}$ And Edwin Perkins ${ }^{1, * *}$ \\ ${ }^{1}$ Department of Mathematics, University of British Columbia, ${ }^{*}$ jlhong@math.ubc.ca; ${ }^{* *}$ perkins@math.ubc.ca \\ ${ }^{2}$ Faculty of Industrial Engineering and Management, Technion, leonid@ie.technion.ac.il
}

We show that if $\partial \mathcal{R}$ is the boundary of the range of super-Brownian motion and dim denotes Hausdorff dimension, then with probability one, for any open set $U, U \cap \partial \mathcal{R} \neq \varnothing$ implies

$$
\operatorname{dim}(U \cap \partial \mathcal{R})= \begin{cases}4-2 \sqrt{2} \approx 1.17 & \text { if } d=2, \\ \frac{9-\sqrt{17}}{2} \approx 2.44 & \text { if } d=3 .\end{cases}
$$

This improves recent results of the last two authors by working with the actual topological boundary, rather than the boundary of the zero set of the local time, and establishing a local result for the dimension.

1. Introduction. We consider a $d$-dimensional super-Brownian motion (SBM), $\left(X_{t}, t \geq\right.$ $0)$, starting at $X_{0}$ under $\mathbb{P}_{X_{0}}$ with $d \leq 3$. Here, $X_{0} \in M_{F}\left(\mathbb{R}^{d}\right)$, the space of finite measures on $\mathbb{R}^{d}$ with the weak topology, $X$ is a continuous $M_{F}\left(\mathbb{R}^{d}\right)$-valued strong Markov process and $\mathbb{P}_{X_{0}}$ denotes any probability under which $X$ is as above. We write $X_{t}(\phi)$ for the integral of $\phi$ with respect to $X$, and take our branching rate to be one, so that for any nonnegative bounded Borel functions $\phi, f$ on $\mathbb{R}^{d}$,

$$
\mathbb{E}_{X_{0}}\left(\exp \left(-X_{t}(\phi)-\int_{0}^{t} X_{s}(f) d s\right)\right)=\exp \left(-X_{0}\left(V_{t}(\phi, f)\right) .\right.
$$

Here, $V_{t}(x)=V_{t}(\phi, f)(x)$ is the unique solution of the mild form of

$$
\frac{\partial V}{\partial t}=\frac{\Delta V_{t}}{2}-\frac{V_{t}^{2}}{2}+f, \quad V_{0}=\phi
$$

that is,

$$
V_{t}=P_{t}(\phi)+\int_{0}^{t} P_{s}\left(f-\frac{V_{t-s}^{2}}{2}\right) d s .
$$

In the above, $\left(P_{t}\right)$ is the semigroup of standard $d$-dimensional Brownian motion. See Chapter II of [15] for the above and further properties. Note that $X$ has an a.s. finite extinction time and, therefore, we can define the so-called total occupation time measure of the superBrownian motion as a finite measure,

$$
I(A)=\int_{0}^{\infty} X_{S}(A) d s .
$$

$\operatorname{Supp}(\mu)$ will denote the closed support of a measure $\mu$. We define the range, $\mathcal{R}$, of $X$ to be

$$
\mathcal{R}=\operatorname{Supp}(I) .
$$

A slightly smaller set is often used in the literature (see [3] or Corollary 9 in Chapter IV of [12]) but the definitions agree under $\mathbb{P}_{\delta_{x}}$ or the canonical measures $\mathbb{N}_{x}$ defined below, and 
also give the same outcomes for $\mathcal{R} \cap \operatorname{Supp}\left(X_{0}\right)^{c}$ and $\partial \mathcal{R} \cap \operatorname{Supp}\left(X_{0}\right)^{c}$. Therefore, the two definitions will be equivalent for our purposes. In dimensions $d \leq 3$, the occupation measure $I$ has a density, $L^{x}$, which is called (total) local time of $X$, that is,

$$
I(f)=\int_{0}^{\infty} X_{s}(f) d s=\int_{\mathbb{R}^{d}} f(x) L^{x} d x \text { for all nonnegative measurable } f .
$$

Moreover, $x \mapsto L^{x}$ is lower semicontinuous, is continuous on $\operatorname{Supp}\left(X_{0}\right)^{c}$, and for $d=1$ is globally continuous (see Theorems 2 and 3 of [18]). From (1.1) and (1.2), it is easy to derive (see Lemma 2.2 in [14])

$$
\mathbb{E}_{X_{0}}\left(e^{-\lambda L^{x}}\right)=\exp \left(-\int_{\mathbb{R}^{d}} V^{\lambda}\left(x-x_{0}\right) X_{0}\left(d x_{0}\right)\right)
$$

where $V^{\lambda}$ is the unique solution (see Section 2 of [14] and the references given there) to

$$
\frac{\Delta V^{\lambda}}{2}=\frac{\left(V^{\lambda}\right)^{2}}{2}-\lambda \delta_{0}, \quad V^{\lambda}>0 \text { on } \mathbb{R}^{d} .
$$

Thus in dimensions $d \leq 3$ we have

$$
\mathcal{R}=\overline{\left\{x: L^{x}>0\right\}}
$$

and $\mathcal{R}$ is a closed set of positive Lebesgue measure. In dimensions $d \geq 4, \mathcal{R}$ is a Lebesgue null set of Hausdorff dimension 4 (see Theorem 1.4 of [3]), which explains our restriction to $d \leq 3$ in this work.

Our main goal in this paper is to study properties of $\partial \mathcal{R}$, the topological boundary of $\mathcal{R}$, and in particular to determine the local Hausdorff dimension of $\partial \mathcal{R}$ outside the support of $X_{0}$. The related question of the dimension of the boundary of the set where the local time is positive, that is, the dimension of

$$
F=\partial\left\{x: L^{x}>0\right\}
$$

was studied in [14]. To describe this latter result, we introduce

$$
p=p(d)= \begin{cases}3 & \text { if } d=1 \\ 2 \sqrt{2} & \text { if } d=2, \\ \frac{1+\sqrt{17}}{2} & \text { if } d=3\end{cases}
$$

$d_{f}=d+2-p$, and

$$
\alpha=\alpha(d)=\frac{p(d)-2}{4-d}= \begin{cases}1 / 3 & \text { if } d=1 \\ \sqrt{2}-1 & \text { if } d=2 \\ \frac{\sqrt{17}-3}{2} & \text { if } d=3\end{cases}
$$

THEOREM 1.1 ([14]). With $\mathbb{P}_{\delta_{0}}$-probability one,

$$
\operatorname{dim}(F)=d_{f}= \begin{cases}0 & \text { if } d=1, \\ 4-2 \sqrt{2} \approx 1.17 & \text { if } d=2, \\ \frac{9-\sqrt{17}}{2} \approx 2.44 & \text { if } d=3 .\end{cases}
$$


There were also versions of the above in [14] for more general initial conditions $X_{0}$.

I. Benjamini's observation that the boundary of the range exhibited interesting fractal properties in simulations was one motivation for the above. Although $F$ may be a natural object from a stochastic analyst's perspective, the topological boundary of $\mathcal{R}, \partial \mathcal{R}$, is the more natural geometric object and of course was the set Benjamini had in mind. Clearly, $\partial \mathcal{R}$ and $F$ are closely related; it is easy to check that

$$
\partial \mathcal{R} \subset F
$$

Thus, Theorem 1.1 gives an upper bound on dimension of $\partial \mathcal{R}$. Whether or not $F=\partial \mathcal{R}$ remains open for $d=2$ or 3, but Theorem 1.7 in [14] shows that, if $d=1$, there exist random variables $L, R$ such that

$$
F=\partial \mathcal{R}=\{\mathrm{L}, \mathrm{R}\} \quad \text { where } \mathrm{L}<0<\mathrm{R} \quad \mathbb{P}_{\delta_{0}} \text {-a.s., }
$$

and so we will usually assume $d=2$ or 3 . A point $x$ in $F$ will be in $\partial \mathcal{R}$ iff there are open sets $U$ approaching $x$ s.t. $L=0$ on $U$. Note that, for example, any isolated zeros of $L$ will be in $F$ but not in $\partial \mathcal{R}$, but we do not even know if such points exist in $d=2,3$. It was conjectured in (1.10) of [14] that in $d=2,3$,

$$
\operatorname{dim}(\partial \mathcal{R})=\operatorname{dim}(F) \quad \mathbb{P}_{\delta_{0}} \text {-a.s. }
$$

In this paper, we verify this conjecture, and prove the following stronger local version.

THEOREM 1.2. $\quad \mathbb{P}_{X_{0}}$-a.s. for any open $U \subset \operatorname{Supp}\left(X_{0}\right)^{c}$,

$$
U \cap \partial \mathcal{R} \neq \varnothing \quad \Rightarrow \quad \operatorname{dim}(U \cap \partial \mathcal{R})=d_{f} .
$$

The following corollary is immediate.

Corollary 1.3. $\mathbb{P}_{X_{0}}$-a.s.

$$
\operatorname{Supp}\left(X_{0}\right)^{c} \cap \partial \mathcal{R} \neq \varnothing \Rightarrow \operatorname{dim}\left(\operatorname{Supp}\left(X_{0}\right)^{c} \cap \partial \mathcal{R}\right)=d_{f} .
$$

The hypothesis in the above corollary is needed; see Proposition 1.5 of [14] for an example where it fails with positive probability.

COROLLARY 1.4. $\quad \mathbb{P}_{\delta_{0}}$-a.s. for any open set $U$,

$$
U \cap \partial \mathcal{R} \neq \varnothing \quad \Rightarrow \quad \operatorname{dim}(U \cap \partial \mathcal{R})=d_{f} .
$$

In particular, $\operatorname{dim}(\partial \mathcal{R})=d_{f} \mathbb{P}_{\delta_{0}}$-a.s.

PRoOF. By Theorem 1.4 and Theorem 1.6 of [7], in $d=2$ and $d=3$ we have $\mathbb{P}_{\delta_{0}}$-a.s. that $L^{x} \rightarrow \infty$ as $x \rightarrow 0$. Therefore, we can conclude that $\mathbb{P}_{\delta_{0}}$-a.s. there is some $\delta>0$ such that $L^{x}>0$ for all $|x|<\delta$ and so $0 \notin \partial \mathcal{R}$, which gives $U \cap \partial \mathcal{R} \neq \varnothing \Rightarrow(U \backslash\{0\}) \cap \partial \mathcal{R} \neq \varnothing$. Now we may apply Theorem 1.2 with $U \backslash\{0\}$ in place of $U$ to complete the proof.

Note that besides confirming (1.10), the above shows that the dimension result holds locally on any open ball intersecting $\partial \mathcal{R}$.

We also consider $X$ and its local time under the canonical measures $\mathbb{N}_{x}$. Recall from Section II.7 of [15] that $\mathbb{N}_{x}$ is a $\sigma$-finite measure on the space of continuous finite length $M_{F}\left(\mathbb{R}^{d}\right)$-valued excursion paths such that

$$
X_{t}=\int v_{t} \Xi(d \nu) \quad \text { for all } t>0 \text { under } \mathbb{P}_{X_{0}}
$$


where $\Xi$ is a Poisson point process with intensity $\mathbb{N}_{X_{0}}(\cdot)=\int \mathbb{N}_{x_{0}}(\cdot) X_{0}\left(d x_{0}\right)$. In this way, $\mathbb{N}_{x_{0}}$ governs the "excursions" of $X$ from a single ancestor at $x_{0}$. The existence of local time $L$ under $\mathbb{N}_{X}$ follows easily from the above, in fact it is even globally continuous (see [7]). It should not be surprising that Corollary 1.4 continues to hold under the canonical measure, in fact, as we shall see, the proof is a bit easier.

THEOREM 1.5. $\mathbb{N}_{0}$-a.e. for any open set $U$,

$$
U \cap \partial \mathcal{R} \neq \varnothing \quad \Rightarrow \quad \operatorname{dim}(U \cap \partial \mathcal{R})=d_{f} .
$$

We first say a few words about the argument leading to the proof of Theorem 1.1 in [14]. If a small ball $B$ intersects $F$, then $B$ contains a point $x$ such that $L^{x}$ is positive but small. Thus, to get the bounds on the Hausdorff dimension of $F$, it is useful to understand the asymptotics of $\mathbb{P}_{\delta_{0}}\left(0<L^{x}<\varepsilon\right)$, as $\varepsilon \downarrow 0$. We write $f(t) \sim g(t)$ as $t \downarrow 0$ iff $f(t) / g(t)$ is bounded and bounded away from zero for small positive $t$, and similarly for $f(t) \sim g(t)$ as $t \uparrow \infty$. It was shown in Theorem 1.3 of [14] that for $p$ as in (1.6) and $\alpha$ given by (1.7),

$$
\mathbb{P}_{\delta_{0}}\left(0<L^{x}<\varepsilon\right) \sim|x|^{-p} \varepsilon^{\alpha} \quad \text { as } \varepsilon \downarrow 0 .
$$

Not very difficult heuristics involving regularity properties of local time and a covering argument explains the upper bound on dimension of $F: \operatorname{dim}(F) \leq d_{f}$ (see the Introduction of [14]). (1.12) was derived in [14] through a Tauberian theorem we now sketch. Let $\lambda \uparrow \infty$ in (1.3) and (1.4) to see that $V^{\lambda}(x) \uparrow V^{\infty}(x)$ where

$$
\mathbb{P}_{\delta_{0}}\left(L^{x}=0\right)=\exp \left(-V^{\infty}(x)\right)
$$

One important simplification available for the analysis of $F$ in [14] is that $V^{\infty}$ is explicitly known (see, e.g., (2.17) in [14]):

$$
V^{\infty}(x)=\frac{2(4-d)}{|x|^{2}} .
$$

In particular, $V^{\infty}$ solves

$$
\frac{\Delta V^{\infty}}{2}=\frac{\left(V^{\infty}\right)^{2}}{2} \text { for } x \neq 0 .
$$

$V^{\infty}$ sometimes is called the very singular solution to (1.15); see, for example, [2]. Applying a Tauberian theorem, one can see that (1.12) can be reduced to verifying

$$
\mathbb{E}_{\delta_{0}}\left(e^{-\lambda L^{x}} 1\left(L^{x}>0\right)\right) \sim|x|^{-p} \lambda^{-\alpha} \text { as } \lambda \uparrow \infty .
$$

The left-hand side of the above behaves like $d^{\lambda}(x):=V^{\infty}(x)-V^{\lambda}(x)$, and so a substantial part of the argument in [14] was devoted to finding a rate of convergence of $V^{\lambda}$ to $V^{\infty}$, and showing that it behaves like the right-hand side of (1.16).

The upper bound on $\operatorname{dim}(F)$ in [14] also utilized Dynkin's exit measures. For nonempty subsets $K_{1}, K_{2}$ of $\mathbb{R}^{d}$, we set

$$
d\left(K_{1}, K_{2}\right):=\inf \left\{|x-y|: x \in K_{1}, y \in K_{2}\right\} .
$$

Define

$$
\begin{aligned}
\mathcal{O}_{X_{0}} \equiv & \left\{\text { open sets } D \text { satisfying } d\left(D^{c}, \operatorname{supp}\left(X_{0}\right)\right)>0\right. \text { and a Brownian } \\
& \text { path starting from any } x \in \partial D \text { will exit } D \text { immediately }\}
\end{aligned}
$$

In what follows, we always assume that $G \in \mathcal{O}_{X_{0}}$.

The exit measure of $X$ from such a $G$ under $\mathbb{P}_{X_{0}}$ or $\mathbb{N}_{X_{0}}$ is denoted by $X_{G}$ (see Chapter $\mathrm{V}$ of [12] for a good introduction to exit measures). $X_{G}$ is a random finite measure supported 
on $\partial G$, which intuitively corresponds to the mass started at $X_{0}$ which is stopped at the instant it leaves $G$. The Laplace functional of $X_{G}$ is given by

$$
\begin{aligned}
\mathbb{E}_{X_{0}}\left(\exp \left(-X_{G}(g)\right)\right) & =\exp \left(-\int 1-\exp \left(-X_{G}(g)\right) d \mathbb{N}_{X_{0}}\right) \\
& =\exp \left(-\int U^{g}(x) X_{0}(d x)\right)
\end{aligned}
$$

where $g: \partial G \rightarrow[0, \infty)$ is continuous and $U^{g} \geq 0$ is the unique continuous function on $\bar{G}$ which is $C^{2}$ on $G$ and solves

$$
\Delta U^{g}=\left(U^{g}\right)^{2} \quad \text { on } G, \quad U^{g}=g \quad \text { on } \partial G .
$$

For this, see Theorem 6 in Chapter V of [12], and the last exercise on page 86 for uniqueness. Let

$$
G_{\varepsilon}^{x_{0}}=G_{\varepsilon}\left(x_{0}\right)=\left\{x:\left|x-x_{0}\right|>\varepsilon\right\} \quad \text { and set } G_{\varepsilon}=G_{\varepsilon}(0) .
$$

Similarly, $B\left(x_{0}, \varepsilon\right)=B_{\varepsilon}\left(x_{0}\right)$ is the open ball centered at $x_{0}$ and $B_{\varepsilon}=B_{\varepsilon}(0)$. $\bar{B}_{\varepsilon}\left(x_{0}\right)$ will denote the closed ball centered at $x_{0}$. Proposition 3.4 of [14] gives an upper bound on $\mathbb{P}_{\delta_{0}}\left(0<X_{G_{\varepsilon}^{x}}(1)<\varepsilon\right)$ as $\varepsilon \downarrow 0$ for $x \neq 0$. This bound is refined to precise asymptotics in Propositions 4.9 and 4.11 in Section 4 below. Intuitively, these asymptotics are related to (1.12) since a small exit measure from $G_{\varepsilon}^{x}$ suggests small values of the local time inside $B_{\varepsilon}(x)$. Estimates for exit measures of small balls are also considered in [1]. There the interest is in the exit measure from a fixed open set and how much mass it puts on a small ball around an exit point on the boundary.

Consider next the ideas underlying Theorem 1.2, where exit measures play a more central role. To show that a point $x$ is near $\partial \mathcal{R}$, it is not enough to show that the local time at $x$ is small and positive, or that the exit measure from some $G_{\varepsilon}^{x}$ is small. In addition, there should be balls $B$ near $x$ on which the local time is zero, or equivalently $X_{\bar{B}^{c}}=0$. To this end, we will study the probability $\mathbb{P}_{\delta_{0}}\left(0<X_{G_{\varepsilon}^{x}}(1) \leq K \varepsilon^{2}, X_{G_{\varepsilon / 2}^{x}}(1)=0\right)$ and show (see Theorem 4.1 and Proposition 4.9)

$$
\mathbb{P}_{\delta_{0}}\left(0<X_{G_{\varepsilon}^{x}}(1) \leq K \varepsilon^{2}, X_{G_{\varepsilon / 2}^{x}}(1)=0\right) \sim \varepsilon^{p-2} \quad \text { as } \varepsilon \downarrow 0 .
$$

The proof of (1.22) requires asymptotics for solutions to (1.20) with varying boundary conditions, rather than solutions to (1.4). For $\varepsilon>0$ and $\lambda \geq 0$, we let $U^{\lambda, \varepsilon}$ denote the unique continuous function on $\{|x| \geq \varepsilon\}$ such that (cf. (1.20))

$$
\Delta U^{\lambda, \varepsilon}=\left(U^{\lambda, \varepsilon}\right)^{2} \quad \text { for }|x|>\varepsilon, \quad \text { and } \quad U^{\lambda, \varepsilon}(x)=\lambda \text { for }|x|=\varepsilon .
$$

Uniqueness of solutions implies the scaling property

$$
U^{\lambda, \varepsilon}(x)=\varepsilon^{-2} U^{\lambda \varepsilon^{2}, 1}(x / \varepsilon) \text { for all }|x| \geq \varepsilon,
$$

and also shows $U^{\lambda, \varepsilon}$ is radially symmetric, thus allowing us to write $U^{\lambda, \varepsilon}(|x|)$ for the value at $x \in \mathbb{R}^{d}$. By (1.19), we have for any finite initial measure satisfying $\operatorname{Supp}\left(X_{0}\right) \subset G_{\varepsilon}$,

$$
\mathbb{E}_{X_{0}}\left(\exp \left(-\lambda X_{G_{\varepsilon}}(1)\right)\right)=\exp \left(-X_{0}\left(U^{\lambda, \varepsilon}\right)\right)
$$

Let $\lambda \uparrow \infty$ in the above to see that $U^{\lambda, \varepsilon} \uparrow U^{\infty, \varepsilon}$ on $G_{\varepsilon}$ and

$$
\mathbb{P}_{X_{0}}\left(X_{G_{\varepsilon}}(1)=0\right)=\exp \left(-X_{0}\left(U^{\infty, \varepsilon}\right)\right) .
$$

Proposition 9(iii) of [12] readily implies (see (3.5) and (3.6) of [14])

$$
U^{\infty, \varepsilon} \text { is } C^{2} \text { and } \Delta U^{\infty, \varepsilon}=\left(U^{\infty, \varepsilon}\right)^{2} \quad \text { on } G_{\varepsilon},
$$

$$
\lim _{|x| \rightarrow \varepsilon,|x|>\varepsilon} U^{\infty, \varepsilon}(x)=+\infty, \quad \lim _{|x| \rightarrow \infty} U^{\infty, \varepsilon}(x)=0 .
$$


Clearly, a key step in deriving (1.22) are asymptotics for

$$
\mathbb{P}_{\delta_{0}}\left(0<X_{G_{\varepsilon}^{x}}(1) \leq K \varepsilon^{2}\right) \sim U^{\infty, \varepsilon}(x)-U^{K^{-1} \varepsilon^{-2}, \varepsilon}(x) \quad \text { as } \varepsilon \downarrow 0,
$$

where the above equivalence is by a Tauberian theorem. In Section 4.1, we show (see Corollary 4.7)

$$
U^{\infty, \varepsilon}(x)-U^{K^{-1} \varepsilon^{-2}, \varepsilon}(x) \sim \varepsilon^{p-2} .
$$

This and a special Markov property (Propositions 2.2 and 2.3) then give (1.22). To get a lower bound on $\partial \mathcal{R}$, essentially by an inclusion-exclusion argument, in addition to the lower bound in (1.22), we will also need an upper bound on (see Proposition 5.1)

$$
\mathbb{P}_{\delta_{0}}\left(0<X_{G_{\varepsilon}^{x_{1}}}(1) \leq K \varepsilon^{2}, 0<X_{G_{\varepsilon}^{x_{2}}}(1) \leq K \varepsilon^{2}\right) .
$$

Although involved, this argument is quite similar to the proof of Proposition 6.1 in [14] and so is omitted (it can be found in Supplementary Material [9]). The above estimates allow us to show that the lower bound on the dimension of $\partial \mathcal{R}$ holds with positive probability; see Proposition 5.3. To conclude the proof of Theorem 1.2, we will show that the lower bound on local dimension, in fact holds with probability one. This will be a consequence of the following proposition.

Proposition 1.6. Let $x_{1} \in \mathbb{R}^{d}, r_{0}>0$, satisfy $B_{2 r_{0}}\left(x_{1}\right) \subset \operatorname{Supp}\left(X_{0}\right)^{c}$. If $r_{1} \in\left(0, r_{0}\right)$, then $\mathbb{P}_{X_{0}}$-a.s.,

$$
X_{G_{r_{1}}^{x_{1}}}(1)=0 \quad \text { and } \quad X_{G_{r_{0}}^{x_{1}}}(1)>0 \quad \text { imply } \quad \operatorname{dim}\left(B_{r_{0}}\left(x_{1}\right) \cap \partial \mathcal{R}\right) \geq d_{f} .
$$

The main ingredient in the proof of Proposition 1.6 is a version under the canonical measure.

Proposition 1.7. Let $x_{1} \in \mathbb{R}^{d}, r_{0}>0$, satisfy $B_{2 r_{0}}\left(x_{1}\right) \subset \operatorname{Supp}\left(X_{0}\right)^{c}$. If $r_{1} \in\left(0, r_{0}\right)$, then $\mathbb{N}_{X_{0}}$-a.e.

$$
\left\{\begin{array}{l}
X_{G_{r_{1}} x_{1}}(1)=0 \text { and } X_{G_{r_{0}}^{x_{1}}}(1)>0 \text { imply } \\
\operatorname{dim}\left(B_{r}\left(x_{1}\right) \cap \partial \mathcal{R}\right) \geq d_{f} \quad \text { for every } r>r_{1} \text { s.t. } X_{G_{r}^{x_{1}}}(1)>0 .
\end{array}\right.
$$

The paper is organized as follows. In Section 2, preliminary results on super-Brownian motion, Brownian snakes, exit measures and their special Markov property are presented. In Section 3, we prove Theorems 1.2 and 1.5, assuming Propositions 1.6, 1.7.

In Section 4, left-tail asymptotics of exit measures are given. First, in Section 4.1 we derive necessary bounds on solutions to the boundary value problems (1.23) and (1.27), and then in Section 4.2 we prove (1.22) (see Theorem 4.1 and Proposition 4.9). In Section 5, we show that the lower bound on the local dimension of $\partial \mathcal{R}$ holds with positive probability; see Proposition 5.3 and Lemma 5.4.

In Section 6, in preparation for the proofs of Propositions 1.6 and 1.7, we analyze the process of exit measures $\left(X_{G_{r}}(1), 0<r \leq r_{0}\right)$ through the rescaled and time-changed process, $Z(t)=X_{G_{r_{0} e^{-t}}}(1) /\left(r_{0} e^{-t}\right)^{2}, t \geq 0$. We refer the reader to Section 6 for the precise definition of a continuous state branching process (CSBP).

Proposition 1.8. Assume $X_{0}$ is a finite initial measure and let $r_{0}>0$ so that $B_{2 r_{0}} \subset$ $\operatorname{Supp}\left(X_{0}\right)^{c}$. Under $\mathbb{N}_{X_{0}},(Z(t), t \geq 0)$ has a cadlag version with only nonnegative jumps, which is a CSBP such that for $0 \leq t_{1}<t_{2}$ and $\lambda \geq 0$,

$$
\mathbb{N}_{X_{0}}\left(\exp \left(-\lambda Z\left(t_{2}\right)\right) \mid Z(s), s \leq t_{1}\right)=\exp \left(-Z\left(t_{1}\right) u\left(t_{2}-t_{1}, \lambda\right)\right)
$$

where $u(t, \lambda)=e^{2 t} U^{\lambda, 1}\left(e^{t}\right)$ for $t, \lambda \geq 0$. 
This will follow from Proposition 6.2(c) in Section 6. The absence of negative jumps in $Z$ is important in the proof of Proposition 1.7, but we also believe that $Z$ and its associated measure-valued process are of independent interest. Some information about the associated branching mechanism $\Psi$ of $Z$ and its Lévy measure are given in Remark 6.3. This complements results on the exit measure process from balls (as opposed to their complements) in [6].

The proofs of Propositions 1.6 and 1.7 are concluded in Section 7. For the proof of Proposition 1.6, one shows that for $r<r_{0}$ sufficiently small there is a single excursion of $X$ (see (1.11)) governed by $\mathbb{N}_{X_{0}}$ that enters $B_{r}$, and thus by the monotonicity of dimension, Proposition 1.6 follows from Proposition 1.7. Proposition 1.7 (with $x_{1}=0$ without loss of generality) is proved by studying the martingale

$$
M_{r}=\mathbb{N}_{x_{0}}\left(\operatorname{dim}\left(B_{r_{0}} \cap \partial \mathcal{R}\right) \geq d_{f} \mid \mathcal{E}_{r}\right), \quad 0 \leq r<r_{0},\left|x_{0}\right|>2 r_{0},
$$

where $\mathcal{E}_{r}$ is the $\sigma$-field generated by the Brownian snake observed inside $G_{r_{0}-r}$ (see Section 2 for a careful definition). In particular, we analyze

$$
M_{r} \text { as } r \uparrow T_{0}=\inf \left\{r: X_{G_{r_{0}-r}}(1)=0\right\} \quad \text { on }\left\{0<T_{0} \leq r_{0}-r_{1}\right\},
$$

where $r_{0}, r_{1}$ are as in Proposition 1.7. The special Markov property and results from Sections 5 and 6 will show $M_{r} \geq q>0$, for $r$ close to $T_{0}$, and on the above set. The last step is to show that $\left\{\operatorname{dim}\left(B_{r_{0}} \cap \partial \mathcal{R}\right) \geq d_{f}\right\} \in \mathcal{E}_{T_{0}-}$ (see Lemma 7.3). Now let $r \uparrow T_{0}$ in (1.31) to see that on $\left\{0<T_{0} \leq r_{0}-r_{1}\right\}, 1\left(\operatorname{dim}\left(B_{r_{0}} \cap \partial \mathcal{R}\right) \geq d_{f}\right) \geq q>0$, as required.

Note that the methods used in [14] (see Theorem 1.4 and the ensuing discussion of that work) would have required the stronger hypothesis $\operatorname{Conv}\left(X_{0}\right)^{c} \cap \partial \mathcal{R} \neq \varnothing$ in Corollary 1.3, where $\operatorname{Conv}\left(X_{0}\right)$ is the closed convex hull of $\operatorname{Supp}\left(X_{0}\right)$. This is because exit measures from hyperplanes were used in [14], instead of the process of exit measure from the complements of shrinking balls. This refinement also leads to the purely local result on dimension in Theorem 1.2.

Convention on functions and constants. Constants whose value is unimportant and may change from line to line are denoted $C, c, c_{d}, c_{1}, c_{2}, \ldots$, while constants whose values will be referred to later and appear initially in say, Lemma i.j are denoted $c_{i . j}$ or $\underline{c}_{i . j}$ or $C_{i . j}$.

\section{Exit measures and the special Markov property.}

Notation. Let $\mathcal{K}$ be the space of compact subsets of $\mathbb{R}^{d}$ equipped with the Hausdorff metric; we add $\varnothing$ as a discrete point. That is, let $K^{\varepsilon}=\{x: d(x, K) \leq \varepsilon\}$ where $d(x, K)=$ $\inf \{|x-y|: y \in K\}$ and for $K_{1}, K_{2}$ nonempty compacts, set

$$
\rho\left(K_{1}, K_{2}\right)=\inf \left\{\varepsilon>0: K_{1} \subset K_{2}^{\varepsilon} \text { and } K_{2} \subset K_{1}^{\varepsilon}\right\} \wedge 1,
$$

and $\rho(\varnothing, K)=1$ for $K$ nonempty compact. $(\mathcal{K}, \rho)$ is then a complete separable metric space. If $U$ is an open set in $\mathbb{R}^{d}$, we let $C(U)$ be the space of continuous functions on $U$ with the compact-open topology.

We start with a measurability result requiring a bit of care; a proof is given in the Appendix.

LEMMA 2.1. (a) For any $R>0$, we have $\psi_{a}: \mathcal{K} \rightarrow \mathcal{K}$ is a Borel map, where $\psi_{a}(K)=$ $K \cap \overline{B_{R}}$.

(b) For any $\alpha, R>0, \psi_{b}: \mathcal{K} \rightarrow \mathbb{R}$ is a universally measurable map, where $\psi_{b}(K)=$ $1\left(\operatorname{dim}\left((\partial K) \cap B_{R}\right)<\alpha\right)$. 
We will use Le Gall's Brownian snake construction of a $\operatorname{SBM} X$, with initial state $X_{0} \in$ $M_{F}\left(\mathbb{R}^{d}\right)$. Set $\mathcal{W}=\bigcup_{t \geq 0} C\left([0, t], \mathbb{R}^{d}\right)$ with the natural metric (see page 54 of [12]), and let $\zeta(w)=t$ be the lifetime of $w \in C\left([0, t], \mathbb{R}^{d}\right) \subset \mathcal{W}$. The Brownian snake $W=\left(W_{t}, t \geq 0\right)$ is a $\mathcal{W}$-valued continuous strong Markov process and, abusing notation slightly, let $\mathbb{N}_{x}$ denote its excursion measure starting from the path at $x \in \mathbb{R}^{d}$ with lifetime zero. As usual, we let $\hat{W}(t)=W_{t}\left(\zeta\left(W_{t}\right)\right)$ denote the tip of the snake at time $t$, and $\sigma(W)>0$ denote the length of the excursion path. We refer the reader to Chapter IV of [12] for the precise definitions. The construction of super-Brownian motion, $X=X(W)$ under $\mathbb{N}_{x}$ or $\mathbb{P}_{X_{0}}$, may be found in Chapter IV of [12]. The "law" of $X(W)$ under $\mathbb{N}_{x}$ is the canonical measure of SBM starting at $x$ described in the last section (and also denoted by $\mathbb{N}_{x}$ ). If $\Xi=\sum_{j \in J} \delta_{W_{j}}$ is a Poisson point process on $\mathcal{W}$ with intensity $\mathbb{N}_{X_{0}}(d W)=\int \mathbb{N}_{x}(d W) X_{0}(d x)$, then by Theorem 4 of Chapter IV of [12] (cf. (1.11))

$$
X_{t}(W)=\sum_{j \in J} X_{t}\left(W_{j}\right)=\int X_{t}(W) \Xi(d W) \text { for } t>0
$$

defines a SBM with initial measure $X_{0}$. We will refer to this as the standard set-up for $X$ under $\mathbb{P}_{X_{0}}$.

Recall $\mathcal{R}=\overline{\left\{x: L^{x}>0\right\}}$ is the range of the SBM $X$ under $\mathbb{P}_{X_{0}}$ or $\mathbb{N}_{X_{0}}$. Under $\mathbb{N}_{X_{0}}$ we have (see (8) on page 69 of [12])

$$
\mathcal{R}=\{\hat{W}(s): s \in[0, \sigma]\}
$$

Let $G \in \mathcal{O}_{X_{0}}$. Then

$X_{G}$ is a finite random measure supported on $\mathcal{R} \cap \partial G$ a.s.

Under $\mathbb{N}_{X_{0}}$, this follows from the definition of $X_{G}$ on page 77 of [12] and the ensuing discussion, and (2.2). Although [12] works under $\mathbb{N}_{x}$ for $x \in G$, the above extends immediately to $\mathbb{P}_{X_{0}}$ because as in (2.23) of [14],

$$
X_{G}=\sum_{j \in J} X_{G}\left(W_{j}\right)=\int X_{G}(W) d \Xi(W),
$$

where $\Xi$ is a Poisson point process on $\mathcal{W}$ with intensity $\mathbb{N}_{X_{0}}$.

Working under $\mathbb{N}_{X_{0}}$ and following [11], we define

$$
\begin{aligned}
S_{G}\left(W_{u}\right) & =\inf \left\{t \leq \zeta_{u}: W_{u}(t) \notin G\right\} \quad(\inf \varnothing=\infty), \\
\eta_{s}^{G}(W) & =\inf \left\{t: \int_{0}^{t} 1\left(\zeta_{u} \leq S_{G}\left(W_{u}\right)\right) d u>s\right\}, \\
\mathcal{E}_{G} & =\sigma\left(W_{\eta_{s}^{G}}, s \geq 0\right) \vee \sigma\left(\left\{\mathbb{N}_{X_{0}}-\text { null sets }\right\}\right),
\end{aligned}
$$

where $s \rightarrow W_{\eta_{s}^{G}}$ is continuous (see page 401 of [11]). Write the open set $\left\{u: S_{G}\left(W_{u}\right)<\zeta_{u}\right\}$ as countable union of disjoint open intervals, $\bigcup_{i \in I}\left(a_{i}, b_{i}\right)$. Clearly, $S_{G}\left(W_{u}\right)=S_{G}^{i}<\infty$ for all $u \in\left[a_{i}, b_{i}\right]$ and we may define

$$
W_{s}^{i}(t)=W_{\left(a_{i}+s\right) \wedge b_{i}}\left(S_{G}^{i}+t\right) \quad \text { for } 0 \leq t \leq \zeta_{\left(a_{i}+s\right) \wedge b_{i}}-S_{G}^{i} .
$$

Therefore, for $i \in I, W^{i} \in C\left(\mathbb{R}_{+}, \mathcal{W}\right)$ are the excursions of $W$ outside $G$. Proposition 2.3 of [11] implies $X_{G}$ is $\mathcal{E}_{G}$-measurable and Corollary 2.8 of the same reference implies

$$
\left\{\begin{array}{l}
\text { Conditional on } \mathcal{E}_{G}, \text { the point measure } \sum_{i \in I} \delta_{W^{i}} \text { is a Poisson } \\
\text { point measure with intensity } \mathbb{N}_{X_{G}} .
\end{array}\right.
$$


If $D$ is an open set in $\mathcal{O}_{X_{0}}$ such that $\bar{G} \subset D$ and $d\left(D^{c}, \bar{G}\right)>0$ (recall (1.17)), then the definition (and existence) of $X_{D}(W)$ applies equally well to each $X_{D}\left(W^{i}\right)$ and it is easy to check that

$$
X_{D}(W)=\sum_{i \in I} X_{D}\left(W^{i}\right)
$$

If $U$ is an open subset of $\operatorname{Supp}\left(X_{0}\right)^{c}$, then $L_{U}$, the restriction of the local time $L^{x}$ to $U$, is in $C(U)$. Here are some simple consequences of (2.5).

Proposition 2.2. (a) Let $G_{1}, G_{2}$ be open sets in $\mathcal{O}_{X_{0}}$ such that $\overline{G_{1}} \subset G_{2}$ and $d\left(G_{2}^{c}, \overline{G_{1}}\right)>0$.

(i) If $\psi_{1}: C\left({\overline{G_{1}}}^{c}\right) \rightarrow[0, \infty)$ is Borel measurable, then

$$
\mathbb{N}_{X_{0}}\left(\psi_{1}\left(L_{\bar{G}_{1}}^{c}\right) \mid \mathcal{E}_{G_{1}}\right)=\mathbb{E}_{X_{G_{1}}}\left(\psi_{1}\left(L_{\bar{G}_{1}}{ }^{c}\right)\right) \text {. }
$$

(ii) If $\psi_{2}: M_{F}\left(\mathbb{R}^{d}\right) \rightarrow[0, \infty)$ is Borel measurable, then

$$
\mathbb{N}_{X_{0}}\left(\psi_{2}\left(X_{G_{2}}\right) \mid \mathcal{E}_{G_{1}}\right)=\mathbb{E}_{X_{G_{1}}}\left(\psi_{2}\left(X_{G_{2}}\right)\right) \text {. }
$$

(b) If $0<R_{2}<R_{1}, d\left(\operatorname{Supp}\left(X_{0}\right), \overline{B_{R_{1}}}\right)>0$, and $\psi_{3}: \mathcal{K} \rightarrow[0, \infty)$ is Borel measurable, then

$$
\mathbb{N}_{X_{0}}\left(\psi_{3}\left(\mathcal{R} \cap \overline{B_{R_{2}}}\right) \mid \mathcal{E}_{G_{R_{1}}}\right)=\mathbb{E}_{X_{G_{R_{1}}}}\left(\psi_{3}\left(\mathcal{R} \cap \overline{B_{R_{2}}}\right)\right)
$$

where $G_{R}$ is as in (1.21).

Proof. (a)(i) is Proposition 2.6(b) of [14]. (a)(ii) follows in a similar manner from (2.5), (2.6) and (2.4).

(b) Define $S: C\left(B_{R_{1}}\right) \rightarrow \mathcal{K}$ by $S(f)=\operatorname{Supp}(f):=\overline{\{x: f(x)>0\}}$, where the closure is taken in all of $\mathbb{R}^{d}$. Then it is easy to see that $S$ is Borel measurable, for example by considering the inverse images of closed balls in $\mathcal{K}$. In addition the map $K \rightarrow \overline{B_{R_{2}}} \cap K$ is measurable on $\mathcal{K}$ by Lemma 2.1 (a). Now observe that $\mathcal{R} \cap \overline{B_{R_{2}}}=S\left(L_{B_{R_{1}}}\right) \cap \overline{B_{R_{2}}}$, and so by the above observations is a measurable function of $L_{B_{R_{1}}}$. Therefore, (b) now follows from (a)(i) with $G_{i}=G_{R_{i}}$.

We will need a version of the above under $\mathbb{P}_{X_{0}}$ as well.

Proposition 2.3. For $X_{0} \in M_{F}\left(\mathbb{R}^{d}\right)$ and an open set $G$ in $\mathcal{O}_{X_{0}}$, let $\Psi$ be a bounded measurable function on $C\left(\bar{G}^{c}\right)$ and $\Phi_{i}, i=0,1$ be bounded measurable functions on $M_{F}\left(\mathbb{R}^{d}\right)$ and $M_{F}\left(\mathbb{R}^{d}\right)^{n}$, respectively. Then:

(a) $\mathbb{E}_{X_{0}}\left(\Phi_{0}\left(X_{G}\right) \Psi(L)\right)=\mathbb{E}_{X_{0}}\left(\Phi_{0}\left(X_{G}\right) \mathbb{E}_{X_{G}}(\Psi(L))\right)$.

(b) (i) Let $D_{i}$ be open sets in $\mathcal{O}_{X_{0}}$, such that $d\left(D_{i}^{c}, \bar{G}\right)>0, \forall 1 \leq i \leq n$. Then

$$
\mathbb{E}_{X_{0}}\left(\Phi_{0}\left(X_{G}\right) \Phi_{1}\left(X_{D_{1}}, \ldots, X_{D_{n}}\right)\right)=\mathbb{E}_{X_{0}}\left(\Phi_{0}\left(X_{G}\right) \mathbb{E}_{X_{G}}\left(\Phi_{1}\left(X_{D_{1}}, \ldots, X_{D_{n}}\right)\right)\right) .
$$

(ii) If $0<R_{2}<R_{1}$ and $d\left(\operatorname{Supp}\left(X_{0}\right)^{c}, \overline{B_{R_{1}}}\right)>0$, then

$$
\mathbb{E}_{X_{0}}\left(\Phi_{0}\left(X_{G_{R_{1}}}\right) 1\left(\mathcal{R} \cap \overline{B_{R_{2}}} \neq \varnothing\right)\right)=\mathbb{E}_{X_{0}}\left(\Phi_{0}\left(X_{G_{R_{1}}}\right) \mathbb{P}_{X_{G_{R_{1}}}}\left(\mathcal{R} \cap \overline{B_{R_{2}}} \neq \varnothing\right)\right) .
$$

ProOF. (a) is Proposition 2.6(c) of [14]. (b)(i) follows by the same reasoning there, using (2.5), (2.4) (the latter for each $D_{i}$, as well as $G$ ), and Proposition 2.2(a)(ii), trivially extended to accommodate $\left(X_{D_{1}}, \ldots, X_{D_{n}}\right)$ in place of $X_{G_{2}}$. (b)(ii) follows from (a), as in the proof of Proposition 2.2(b). 
3. Proofs of Theorems 1.2 and 1.5. We will see in this section that (using the upper bound on $\operatorname{dim}(F)$ in Theorem 1.1) Theorem 1.2 is a simple consequence of Proposition 1.6, and similarly Theorem 1.5 can be derived from Proposition 1.7.

Proof of TheOREM 1.2. Let $x_{1} \in \mathbb{R}^{d}$ and let $0<r_{1}<r_{0} \leq 1$ such that $B_{2 r_{0}}\left(x_{1}\right) \subset$ $\operatorname{Supp}\left(X_{0}\right)^{c}$. From (2.3), we have $\mathbb{P}_{X_{0}}$-a.s.,

$$
\partial G_{r_{1}}^{x_{1}} \cap \mathcal{R}=\varnothing \quad \Rightarrow \quad X_{G_{r_{1}}^{x_{1}}}=0 .
$$

Proposition 2.3(b)(ii) and translation invariance imply

$$
\begin{aligned}
& \mathbb{P}_{X_{0}}\left(X_{G_{r_{0}}}^{x_{1}}=0, \mathcal{R} \cap B_{r_{0} / 2}\left(x_{1}\right) \neq \varnothing\right) \\
& \quad=\mathbb{E}_{X_{0}}\left(1\left(X_{G_{r_{0}}^{x_{1}}}=0\right) \mathbb{P}_{X_{G_{r_{0}}}^{x_{1}}}\left(\mathcal{R} \cap B_{r_{0} / 2}\left(x_{1}\right) \neq \varnothing\right)\right)=0 .
\end{aligned}
$$

It follows that $\mathbb{P}_{X_{0}}$-a.s.,

$$
\mathcal{R} \cap B_{r_{0} / 2}\left(x_{1}\right) \neq \varnothing \quad \Rightarrow \quad X_{G_{r_{0}}^{x_{1}}}(1)>0 .
$$

Fix $\omega$ outside a $\mathbb{P}_{X_{0}}$-null set so that (1.29) of Proposition 1.6, (3.1), and (3.2) all hold for all $x_{1} \in \mathbb{Q}^{d}$ and all rational numbers $0<r_{1}<r_{0} \leq 1$ satisfying $B_{2 r_{0}}\left(x_{1}\right) \subset \operatorname{Supp}\left(X_{0}\right)^{c}$. Assume $U$ is an open set in $\operatorname{Supp}\left(X_{0}\right)^{c}$ which intersects $\partial \mathcal{R}$ and choose $x_{0} \in U \cap \partial \mathcal{R}$. Pick a rational $r_{0}$ in $(0,1]$ so that

$$
B_{3 r_{0}}\left(x_{0}\right) \subset U \subset \operatorname{Supp}\left(X_{0}\right)^{c},
$$

then choose $x_{1} \in \mathbb{Q}^{d} \cap B_{r_{0} / 2}\left(x_{0}\right) \cap \mathcal{R}^{c}$, and finally select a rational $r_{1} \in\left(0, r_{0}\right)$ such that

$$
B_{2 r_{1}}\left(x_{1}\right) \subset \mathcal{R}^{c} \quad \text { and so } \quad \partial G_{r_{1}}^{x_{1}} \cap \mathcal{R}=\varnothing .
$$

Clearly, we have

$$
B_{2 r_{0}}\left(x_{1}\right) \subset B_{3 r_{0}}\left(x_{0}\right) \subset U \subset \operatorname{Supp}\left(X_{0}\right)^{c}
$$

and

$$
x_{0} \in B_{r_{0} / 2}\left(x_{1}\right) \cap \partial \mathcal{R} \quad \text { and so } \quad \mathcal{R} \cap B_{r_{0} / 2}\left(x_{1}\right) \neq \varnothing .
$$

Our choice of $\omega$ and (3.4) allow us to conclude from (3.1), (3.3), (3.2) and (3.5), respectively, that

$$
X_{G_{r_{1}}}^{x_{1}(1)=0} \text { and } X_{G_{r_{0}}^{x_{1}}}(1)>0, \quad \text { respectively. }
$$

By (3.4) and our choice of $\omega$ we may also apply Proposition 1.6 with (3.6) and conclude that

$$
\operatorname{dim}(U \cap \partial \mathcal{R}) \geq \operatorname{dim}\left(B_{r_{0}}\left(x_{1}\right) \cap \partial \mathcal{R}\right) \geq d_{f},
$$

where we have used (3.4) in the first inequality. On the other hand, we know from Theorem 1.4(a) of [14] and $\partial \mathcal{R} \subset \partial\left\{x: L^{x}>0\right\}$ that

$$
\operatorname{dim}(U \cap \partial \mathcal{R}) \leq \operatorname{dim}\left(\operatorname{Supp}\left(X_{0}\right)^{c} \cap \partial \mathcal{R}\right) \leq d_{f},
$$

and the proof is complete.

Proof of Theorem 1.5. As in the above proof of Theorem 1.2 from Proposition 1.6, we can derive from Proposition 1.7 that $\mathbb{N}_{X_{0}}$-a.e. for any open set $U \subset \operatorname{Supp}\left(X_{0}\right)^{c}$,

$$
U \cap \partial \mathcal{R} \neq \varnothing \quad \Rightarrow \quad \operatorname{dim}(U \cap \partial \mathcal{R})=d_{f} .
$$

Now we turn to the $\mathbb{N}_{0}$ case in Theorem 1.5. By (4.4) in [8], we have $\mathbb{N}_{0}$-a.e. that $L^{0}>0$. Although the arguments in [8] were given for $d=1$, they work in any dimension $d \leq 3$. Since $L^{x}$ is globally continuous under $\mathbb{N}_{0}$ by Theorem 1.2 of [7], we can conclude that $\mathbb{N}_{0}$-a.e. there is some $\delta>0$ such that $L^{x}>0$ for all $|x|<\delta$, and so $0 \notin \partial \mathcal{R}$, which gives $U \cap \partial \mathcal{R} \neq \varnothing \Rightarrow U \backslash\{0\} \cap \partial \mathcal{R} \neq \varnothing$. Now we may apply (3.7) with $U \backslash\{0\}$ in place of $U$ to complete the proof. 
4. Lower bound on the exit measure probability. Throughout this section, we fix $\varepsilon_{0} \in$ $(0,1)$. As noted in the Introduction, the goal of this section, stated below, is a key estimate for the lower bound on the dimension of $\partial \mathcal{R}$. Although we are interested in $d=2,3$, we assume $d \leq 3$ throughout this section as the arguments remain valid.

THEOREM 4.1. There are positive constants $R_{4.1}, K_{1}\left(\varepsilon_{0}\right)<K_{2}\left(\varepsilon_{0}\right)<\infty$ and $c_{4.1}\left(\varepsilon_{0}\right)$ such that, for all $\varepsilon_{0} \leq|x| \leq \varepsilon_{0}^{-1}$,

$$
\mathbb{P}_{\delta_{x}}\left(K_{1} \leq \frac{X_{G_{\varepsilon}}(1)}{\varepsilon^{2}} \leq K_{2}, X_{G_{\varepsilon / 2}(1)}=0\right) \geq c_{4.1}\left(\varepsilon_{0}\right) \varepsilon^{p-2} \quad \forall 0<\varepsilon<\varepsilon_{0} / R_{4.1} .
$$

The next subsection is devoted to proving necessary bounds on solutions to the boundary value problems (1.23), (1.27). These bounds will be used for proving Theorem 4.1 in Section 4.2.

4.1. Bounds on solutions to some boundary value problems. Recall $U^{\lambda, R}$ and $U^{\infty, R}$ from (1.23) and (1.27), respectively. A simple application of (1.27), (1.15) and the maximum principle implies

$$
V^{\infty}(x) \leq U^{\infty, 1}(x) \quad \forall|x|>1 .
$$

We will need an upper bound on $U^{\infty, 1}$ which shows this bound is asymptotically sharp for large $|x|$. We briefly include $d=1$ in our analysis. Recall that $p$ is as in (1.6).

Proposition 4.2. There exist constants $C_{4.2}>1$ and $c_{4.2} \geq 0$ such that

$$
U^{\infty, 1}(x) \leq V^{\infty}(x)\left(1+c_{4.2}|x|^{2-p}\right) \quad \forall|x| \geq C_{4.2} .
$$

PROOF. We will write $u(r)$ for $U^{\infty, 1}(r)$ and $v(r)$ for $V^{\infty}(r)$.

Let

$$
\begin{aligned}
& q(t)=\frac{u\left(e^{t / 4}\right)}{v\left(e^{t / 4}\right)}=\frac{1}{4} u\left(e^{t / 4}\right) e^{t / 2}, \quad t \geq 0, \text { in } d=2, \\
& q(t)=\frac{u\left(e^{t / 3}\right)}{v\left(e^{t / 3}\right)}=\frac{1}{2} u\left(e^{t / 3}\right) e^{2 t / 3}, \quad t \geq 0, \text { in } d=3, \\
& q(t)=\frac{u\left(e^{t / 5}\right)}{v\left(e^{t / 5}\right)}=\frac{1}{6} u\left(e^{t / 5}\right) e^{2 t / 5}, \quad t \geq 0, \text { in } d=1 .
\end{aligned}
$$

Consider first $d=2$. A simple calculation gives

$$
\begin{aligned}
\frac{1}{2} q^{\prime \prime}-\frac{1}{2} q^{\prime}+\beta\left(q-q^{2}\right) & =0 \quad \text { on }(0, \infty), \\
q(0) & =\infty, \quad \lim _{t \rightarrow \infty} q(t)=1,
\end{aligned}
$$

where $\beta=\frac{1}{8}$ in $d=2$ (similarly $\beta=\frac{3}{25}$ in $d=1$ and $\beta=\frac{1}{9}$ in $d=3$ ). Note that $q(0)=\infty$ by the definitions of $U^{\infty, 1}$ and $V^{\infty}$. Also note that the last limit is derived the same way as (3.10) in [14] by taking $U^{\infty, 1}$ instead of $U^{\delta_{0}, 1}, \tilde{y}(t)=y(t+2)$ instead of $y(t)$ and $\tilde{z}(t)=z(t+2)$ instead of $z(t)$ there.

Note that $q(x) \geq 1$ for all $x \geq 0$ by (4.1). Define

$$
z=q-1,
$$


and thus by the above $z(x) \geq 0$ for all $x \geq 0$. Then $z$ satisfies the following equation:

$$
\frac{1}{2} z^{\prime \prime}-\frac{1}{2} z^{\prime}-\beta z(z+1)=0 \quad \text { on }(0, \infty), \quad z(0)=\infty, \quad \lim _{t \rightarrow \infty} z(t)=0 .
$$

Let $w$ be the unique solution to

$$
\begin{aligned}
\frac{1}{2} w^{\prime \prime}(t)-\frac{1}{2} w^{\prime}(t)-\beta w(t) & =0, \quad t>1, \\
w(1) & =z(1), \quad \lim _{t \rightarrow \infty} w(t)=0 .
\end{aligned}
$$

By the comparison principle and using $z+1 \geq 1$, we get

$$
w(t) \geq z(t), \quad t \geq 1
$$

We leave it for the reader to check that

$$
w(t)=z(1) e^{-\lambda} e^{\lambda t}, \quad t \geq 1,
$$

with

$$
\lambda=\frac{1}{2}-\sqrt{\frac{1}{4}+2 \beta}<0 .
$$

By the definition of $\beta$, we have $\lambda=1 / 2-\frac{1}{\sqrt{2}}$ in $d=2$ (similarly, $\lambda=-0.2$ for $d=1$ and $\lambda=1 / 2-\frac{\sqrt{17}}{6}$ for $d=3$ ). This and (4.5) imply that for $C=z(1) e^{-\lambda} \geq 0$ we have

$$
z(x) \leq C e^{\lambda x}, \quad x \geq 1,
$$

and since $\lambda<0$ we get that $z$ decreases to zero exponentially fast. Recall the definition of $q$ to get

$$
q(x) \leq 1+C e^{\lambda x}, \quad x \geq 1
$$

Then (as $d=2$ ) we have

$$
u\left(e^{t / 4}\right) \leq v\left(e^{t / 4}\right)\left(1+C e^{\lambda t}\right), \quad t \geq 1,
$$

and so

$$
u(s) \leq v(s)\left(1+C s^{4 \lambda}\right)=v(s)\left(1+C s^{2-p}\right), \quad s \geq e^{1 / 4} .
$$

Similar algebra shows the result in $d=1,3$.

Recalling (1.14) and that $p>2$, we may immediately conclude the following.

COROLLARY 4.3. There are constants $C_{4.3}, c_{4.3}>0$ such that for all $x \in \mathbb{R}^{d}$, with $|x| \geq$ $C_{4.3}$, we have

$$
U^{\infty, 1}(x) \leq V^{\infty}(x)+\frac{c_{4.3}}{|x|^{p}} .
$$

In particular, there is some constant $K_{4.3}>2$ such that

$$
U^{\infty, 1}(x) \leq 3(4-d)|x|^{-2} \quad \forall|x| \geq K_{4.3} .
$$


If $D^{\lambda}=U^{\infty, 1}-U^{\lambda, 1} \geq 0$ for $\lambda>0$, then the Feynmann-Kac formula (as in (3.8) in [14]) easily gives

$$
\begin{aligned}
D^{\lambda}(x) & =D^{\lambda}(R) E_{x}\left(1_{\left(\tau_{R}<\infty\right)} \exp \left(-\int_{0}^{\tau_{R}}\left(\frac{U^{\infty, 1}+U^{\lambda, 1}}{2}\right)\left(B_{s}\right) d s\right)\right), \\
|x| \geq R>1 &
\end{aligned}
$$

where $B$ denote a $d$-dimensional Brownian motion starting at $x$ under $P_{x}$ and $\tau_{R}=\inf \{t \geq$ $\left.0:\left|B_{t}\right| \leq R\right\}$ for $|x| \geq R>1$.

We will frequently use the following lemmas. For $\gamma \in \mathbb{R}$, let $\left(\rho_{t}\right)$ denote a $\gamma$-dimensional Bessel process starting from $r>0$ under $P_{r}^{(\gamma)}$. For $R>0$, let $\tau_{R}=\inf \left\{t \geq 0: \rho_{t} \leq R\right\}$. The following result is from Lemma 5.3 of [14].

LEMMA 4.4. Assume $0<2 \gamma \leq v^{2}$ and $q>2$. Then

$$
\sup _{r \geq 1} E_{r}^{(2+2 v)}\left(\exp \left(\int_{0}^{\tau_{1}} \frac{\gamma}{\rho_{s}^{q}} d s\right) \mid \tau_{1}<\infty\right) \leq c_{4.4}(q, v)<\infty .
$$

LEMMA 4.5. Let $q>2, a \in \mathbb{R}, \zeta \in[0,2(4-d)), v_{\zeta}=\sqrt{v^{2}-\zeta}$ and $p_{\zeta}=v_{\zeta}+\mu$, where

$$
\mu=\left\{\begin{array}{ll}
-1 / 2 & \text { if } d=1, \\
0 & \text { if } d=2, \\
1 / 2 & \text { if } d=3
\end{array} \text { and } \quad v=\sqrt{\mu^{2}+4(4-d)}\right.
$$

Then for all $R<|x|$,

$$
\begin{aligned}
& E_{x}\left(1\left(\tau_{R}<\infty\right) \exp \left(\int_{0}^{\tau_{R}} \frac{a}{\left|B_{s}\right|^{q}} d s\right) \exp \left(-\int_{0}^{\tau_{R}} \frac{2(4-d)-\zeta / 2}{\left|B_{S}\right|^{2}} d s\right)\right) \\
& =E_{|x|}^{\left(2+2 v_{\zeta}\right)}\left(\exp \left(\int_{0}^{\tau_{R}} \frac{a}{\rho_{s}^{q}} d s\right) \mid \tau_{R}<\infty\right)(R /|x|)^{p_{\zeta}} .
\end{aligned}
$$

PROOF. The proof is based on arguments from [14] (see the proof of Lemma 5.4 there), which in turn go back to Marc Yor [20], and is deferred to Appendix A.2.

Proposition 4.6. There are positive universal constants $C_{4.6}, c_{4.6}>0, K_{4.6}>K_{4.3}$, and $R_{4.6}>2$ such that:

(a)

$$
D^{\lambda}(x) \leq \frac{R^{p}}{|x|^{p}} D^{\lambda}(R) \quad \forall|x| \geq R>1, \lambda \geq 2(4-d) .
$$

(b)

$$
D^{\lambda}(x) \leq C_{4.6} \frac{R^{p}}{|x|^{p}} D^{\lambda}(R) \quad \forall|x| \geq R \geq \frac{K_{4.6}}{\lambda}, 0<\lambda<1 .
$$

(c)

$$
D^{\lambda}(x) \geq c_{4.6} \frac{R^{p}}{|x|^{p}} D^{\lambda}(R)>0 \quad \forall|x| \geq R \geq R_{4.6}, \lambda>0 .
$$


PROOF. Recall $\mu, v$ introduced in (4.9) so that for $p=p(d)$ defined as in (1.6), we have

$$
p=\mu+v .
$$

(a) For $\lambda \geq 2(4-d)$, clearly we have $U^{\lambda, 1}(1)=\lambda \geq 2(4-d)=V^{\infty}(1)$. As

$$
\lim _{|x| \rightarrow \infty} U^{\lambda, 1}(x) \leq \lim _{|x| \rightarrow \infty} U^{\infty, 1}(x)=0
$$

by (1.27), we may apply the maximum principle to get

$$
U^{\lambda, 1}(x) \geq V^{\infty}(x)=\frac{2(4-d)}{|x|^{2}} \quad \forall|x|>1 .
$$

Use (4.1) and the above to see that (4.8) becomes

$$
D^{\lambda}(x) \leq D^{\lambda}(R) E_{x}\left(1_{\left(\tau_{R}<\infty\right)} \exp \left(-\int_{0}^{\tau_{R}} \frac{2(4-d)}{\left|B_{S}\right|^{2}} d s\right)\right)=D^{\lambda}(R)(R /|x|)^{p},
$$

the last by Lemma 4.5.

(b) Assume $\lambda \in(0,1)$. Recall Proposition 3.3(b) in [14]:

$$
\begin{aligned}
& \forall \delta \in(0,1), \exists C_{\delta}>2, \\
& \quad \text { so that } U^{\lambda, 1}(x) \geq(1-\delta) V^{\infty}(x) \text { for all }|x| \geq C_{\delta} / \lambda .
\end{aligned}
$$

For any $\delta \in(0,1)$, let $\zeta=2(4-d) \delta \in(0,2(4-d))$. Let $\mu$ and $v$ be as in (4.9). Define $v_{\zeta}=$ $\sqrt{\mu^{2}+4(4-d)-\zeta}$ and $p_{\zeta}=v_{\zeta}+\mu \rightarrow p>2$ as $\zeta \downarrow 0$. Choose $\delta \in(0,1)$ small enough so that $p_{\zeta}>2$. Let $K_{4.6} \equiv C_{\delta}+K_{4.3}$. Now use (4.1), (4.13) and Lemma 4.5 to see that for $|x| \geq R \geq K_{4.6} / \lambda>C_{\delta} / \lambda$, (4.8) implies

$$
\begin{aligned}
D^{\lambda}(x) & \leq D^{\lambda}(R) E_{x}\left(1\left(\tau_{R}<\infty\right) \exp \left(-\int_{0}^{\tau_{R}} \frac{2(4-d)-(\zeta / 2)}{\left|B_{S}\right|^{2}} d s\right)\right) \\
& =D^{\lambda}(R)(R /|x|)^{p_{\zeta}} .
\end{aligned}
$$

Let $\xi(R)=D^{\lambda}(R) R^{p_{\zeta}} / 2$. Then the above gives

$$
\left(\frac{U^{\lambda, 1}+U^{\infty, 1}}{2}\right)(x) \geq U^{\infty, 1}(x)-\frac{\xi(R)}{|x|^{p_{\zeta}}} \geq V^{\infty}(x)-\frac{\xi(R)}{|x|^{p_{\zeta}}} \quad \text { for }|x| \geq R .
$$

Use this in (4.8) and then Lemma 4.5 to see that for $|x| \geq R$,

$$
\begin{aligned}
D^{\lambda}(x) & \leq D^{\lambda}(R) E_{x}\left(1\left(\tau_{R}<\infty\right) \exp \left(\int_{0}^{\tau_{R}} \frac{\xi(R)}{\left|B_{S}\right|^{p_{\zeta}}} d s\right) \exp \left(-\int_{0}^{\tau_{R}} \frac{2(4-d)}{\left|B_{S}\right|^{2}} d s\right)\right) \\
& =D^{\lambda}(R) E_{|x|}^{(2+2 v)}\left(\exp \left(\int_{0}^{\tau_{R}} \frac{\xi(R)}{\rho_{s}^{p_{\zeta}}} d s\right) \mid \tau_{R}<\infty\right)(R /|x|)^{p} .
\end{aligned}
$$

A scaling argument shows that the above equals

$$
D^{\lambda}(R)(R /|x|)^{p} E_{|x| / R}^{(2+2 v)}\left(\exp \left(\int_{0}^{\tau_{1}} \frac{\xi(R) R^{2-p_{\zeta}}}{\rho_{s}^{p_{\zeta}}} d s\right) \mid \tau_{1}<\infty\right) .
$$

To apply Lemma 4.4, we note that by (4.7), for $R \geq K_{4.6} / \lambda>K_{4.3}$ we have

$$
2 \gamma \equiv 2 \xi(R) R^{2-p_{\zeta}} \leq U^{\infty, 1}(R) R^{2} \leq 3(4-d)<v^{2} .
$$

So Lemma 4.4 and the above bound show that

$$
D^{\lambda}(x) \leq D^{\lambda}(R)(R /|x|)^{p} c_{4.4}\left(p_{\zeta}, v\right) .
$$


(c) Use (4.6) in Corollary 4.3 to see that for $|x| \geq R>C_{4.3}$, we have

$$
\frac{U^{\infty, 1}+U^{\lambda, 1}}{2}(x) \leq U^{\infty, 1}(x) \leq \frac{2(4-d)}{|x|^{2}}+\frac{c_{4.3}}{|x|^{p}} .
$$

So (4.8) and Lemma 4.5 imply

$$
\begin{aligned}
D^{\lambda}(x) & \geq D^{\lambda}(R) E_{x}\left(1_{\left(\tau_{R}<\infty\right)} \exp \left(-\int_{0}^{\tau_{R}} \frac{c_{4.3}}{\left|B_{s}\right|^{p}} d s\right) \exp \left(-\int_{0}^{\tau_{R}} \frac{2(4-d)}{\left|B_{s}\right|^{2}} d s\right)\right) \\
& =D^{\lambda}(R) E_{|x|}^{(2+2 v)}\left(\exp \left(-\int_{0}^{\tau_{R}} \frac{c_{4.3}}{\rho_{s}^{p}} d s\right) \mid \tau_{R}<\infty\right)(R /|x|)^{p},
\end{aligned}
$$

with $p=\mu+v$. A scaling argument shows that the above equals

$$
D^{\lambda}(R)(R /|x|)^{p} E_{|x| / R}^{(2+2 v)}\left(\exp \left(-\int_{0}^{\tau_{1}} \frac{c_{4.3} R^{2-p}}{\rho_{s}^{p}} d s\right) \mid \tau_{1}<\infty\right) .
$$

To apply Lemma 4.4, note that if $R \geq R_{4.6}$ for some constant $R_{4.6}>2$,

$$
2 \gamma \equiv 2 c_{4.3} R^{2-p}<2(4-d)<v^{2} .
$$

By Cauchy-Schwarz, we have

$$
\begin{aligned}
1= & \left(E_{|x| / R}^{(2+2 v)}\left(\exp \left(-\int_{0}^{\tau_{1}} \frac{c_{4.3} R^{2-p}}{2 \rho_{s}^{p}} d s\right) \exp \left(\int_{0}^{\tau_{1}} \frac{c_{4.3} R^{2-p}}{2 \rho_{s}^{p}} d s\right) \mid \tau_{1}<\infty\right)\right)^{2} \\
\leq & E_{|x| / R}^{(2+2 v)}\left(\exp \left(-\int_{0}^{\tau_{1}} \frac{c_{4.3} R^{2-p}}{\rho_{s}^{p}} d s\right) \mid \tau_{1}<\infty\right) \\
& \times E_{|x| / R}^{(2+2 v)}\left(\exp \left(\int_{0}^{\tau_{1}} \frac{c_{4.3} R^{2-p}}{\rho_{s}^{p}} d s\right) \mid \tau_{1}<\infty\right) \\
\leq & E_{|x| / R}^{(2+2 v)}\left(\exp \left(-\int_{0}^{\tau_{1}} \frac{c_{4.3} R^{2-p}}{\rho_{s}^{p}} d s\right) \mid \tau_{1}<\infty\right) c_{4.4}(p, v),
\end{aligned}
$$

the last by Lemma 4.4. Hence

$$
\inf _{|x| \geq R} E_{|x| / R}^{(2+2 v)}\left(\exp \left(-\int_{0}^{\tau_{1}} \frac{c_{4.3} R^{2-p}}{\rho_{s}^{p}} d s\right) \mid \tau_{1}<\infty\right) \geq c_{4.4}(p, v)^{-1}>0,
$$

and by (4.15) we are done.

By using the scaling relations of $U^{\infty, \varepsilon}$ and $U^{\lambda \varepsilon^{-2}, \varepsilon}$ from (1.24), the following is immediate from the above.

COROLlary 4.7. For all $\varepsilon>0$, we have (a)

$$
U^{\infty, \varepsilon}(x)-U^{\lambda \varepsilon^{-2}, \varepsilon}(x) \leq \frac{R^{p}}{|x|^{p}} D^{\lambda}(R) \varepsilon^{p-2} \quad \forall|x| / \varepsilon \geq R>1, \lambda \geq 2(4-d) .
$$

(b)

$$
U^{\infty, \varepsilon}(x)-U^{\lambda \varepsilon^{-2}, \varepsilon}(x) \leq C_{4.6} \frac{R^{p}}{|x|^{p}} D^{\lambda}(R) \varepsilon^{p-2} \quad \forall|x| / \varepsilon \geq R \geq \frac{K_{4.6}}{\lambda}, 0<\lambda<1 .
$$

(c)

$$
U^{\infty, \varepsilon}(x)-U^{\lambda \varepsilon^{-2}, \varepsilon}(x) \geq c_{4.6} \frac{R^{p}}{|x|^{p}} D^{\lambda}(R) \varepsilon^{p-2}>0 \quad \forall|x| / \varepsilon \geq R \geq R_{4.6}, \lambda>0 .
$$




\subsection{The left tail of the total exit measure and proof of Theorem 4.1.}

Proposition 4.8. For any $|x| \geq \varepsilon_{0}$ and $\varepsilon \in\left(0, \varepsilon_{0} / 2\right)$, we have

$$
\mathbb{P}_{\delta_{x}}\left(0<\frac{X_{G_{\varepsilon}}(1)}{\varepsilon^{2}} \leq \frac{1}{\lambda}\right) \leq e \frac{2^{p}}{|x|^{p}} D^{\lambda}(2) \varepsilon^{p-2} \quad \forall \lambda \geq 2(4-d) .
$$

ProOF. Apply Markov's inequality to get

$$
\begin{aligned}
\mathbb{P}_{\delta_{x}}\left(0<\frac{X_{G_{\varepsilon}}(1)}{\varepsilon^{2}} \leq \frac{1}{\lambda}\right) & \leq e \mathbb{E}_{\delta_{x}}\left(\exp \left(-\lambda \varepsilon^{-2} X_{G_{\varepsilon}}(1)\right) 1\left(X_{G_{\varepsilon}}(1)>0\right)\right) \\
& =e\left(\exp \left(-U^{\lambda \varepsilon^{-2}, \varepsilon}(x)\right)-\exp \left(-U^{\infty, \varepsilon}(x)\right)\right) \\
& \leq e\left(U^{\infty, \varepsilon}(x)-U^{\lambda \varepsilon^{-2}, \varepsilon}(x)\right),
\end{aligned}
$$

the equality by (1.25) and (1.26). Note we've assumed $|x| / \varepsilon \geq \varepsilon_{0} / \varepsilon>2$ and $\lambda \geq 2(4-d$ ) so that we can use Corollary 4.7(a) with $R=2$ to bound the right-hand side of (4.16) by $e(2 /|x|)^{p} D^{\lambda}(2) \varepsilon^{p-2}$, as required.

Proposition 4.9. There is some $c_{4.9}\left(\varepsilon_{0}\right)>0$ such that for all $|x| \geq \varepsilon_{0}$ and $\varepsilon \in\left(0, \varepsilon_{0}\right)$,

$$
\mathbb{P}_{\delta_{x}}\left(0<\frac{X_{G_{\varepsilon}}(1)}{\varepsilon^{2}} \leq \frac{1}{\lambda}\right) \leq c_{4.9}\left(\varepsilon_{0}\right) \lambda^{-(p-2)} \varepsilon^{p-2} \quad \forall 0<\lambda<1 .
$$

ProOF. For $\lambda \in(0,1)$ such that

$$
|x| / \varepsilon \geq \varepsilon_{0} / \varepsilon \geq K_{4.6} / \lambda,
$$

we apply Markov's inequality as in (4.16) and use Corollary 4.7(b) with $R=K_{4.6} / \lambda$ to get

$$
\begin{aligned}
\mathbb{P}_{\delta_{x}}\left(0<\frac{X_{G_{\varepsilon}}(1)}{\varepsilon^{2}} \leq \frac{1}{\lambda}\right) & \leq e\left(U^{\infty, \varepsilon}(x)-U^{\lambda \varepsilon^{-2}, \varepsilon}(x)\right) \\
& \leq e C_{4.6} U^{\infty, 1}(R)(R /|x|)^{p} \varepsilon^{p-2} \\
& \leq e C_{4.6}\left(3(4-d) / R^{2}\right)\left(R / \varepsilon_{0}\right)^{p} \varepsilon^{p-2} \\
& \leq 9 e C_{4.6} \varepsilon_{0}^{-p} K_{4.6}^{p-2} \lambda^{-(p-2)}{ }_{\varepsilon}^{p-2},
\end{aligned}
$$

the second last inequality by (4.7) with $R=K_{4.6} / \lambda>K_{4.6}>K_{4.3}$.

For $\lambda \in(0,1)$ such that

$$
\varepsilon_{0} / \varepsilon \leq K_{4.6} / \lambda
$$

we have

$$
\mathbb{P}_{\delta_{x}}\left(0<\frac{X_{G_{\varepsilon}}(1)}{\varepsilon^{2}} \leq \frac{1}{\lambda}\right) \leq 1 \leq\left(K_{4.6} / \varepsilon_{0}\right)^{p-2} \lambda^{-(p-2)} \varepsilon^{p-2}
$$

The result follows by letting $c_{4.9}\left(\varepsilon_{0}\right)=9 e C_{4.6} \varepsilon_{0}^{-p} K_{4.6}^{p-2}+\left(K_{4.6} / \varepsilon_{0}\right)^{p-2}$.

For $|x| \geq \varepsilon_{0}$ and $\varepsilon \in\left(0, \varepsilon_{0}\right)$, we define

$$
F_{\varepsilon, x}(K) \equiv \mathbb{P}_{\delta_{x}}\left(0<\frac{X_{G_{\varepsilon}}(1)}{\varepsilon^{2}} \leq K\right) \quad \forall K>0
$$

and

$$
\hat{F}_{\varepsilon, x}(\lambda) \equiv \mathbb{E}_{\delta_{x}}\left(\exp \left(-\lambda \frac{X_{G_{\varepsilon}}(1)}{\varepsilon^{2}}\right) 1\left(X_{G_{\varepsilon}}(1)>0\right)\right) \quad \forall \lambda>0 .
$$

The dependence on $\varepsilon$ and $x$ will at times be suppressed. 
LEMMA 4.10. There are constants $R_{4.10}>2$ and $c_{4.10}\left(\varepsilon_{0}\right)>0$ so that for any $\varepsilon_{0} \leq|x| \leq$ $\varepsilon_{0}^{-1}$ and $\varepsilon \in\left(0, \varepsilon_{0} / R_{4.10}\right)$,

$$
\mathbb{E}_{\delta_{x}}\left(\exp \left(-\lambda \frac{X_{G_{\varepsilon}}(1)}{\varepsilon^{2}}\right) 1\left(X_{G_{\varepsilon}}(1)>0\right)\right) \geq c_{4.10}\left(\varepsilon_{0}\right) D^{\lambda}\left(R_{4.10}\right) \varepsilon^{p-2} \quad \forall \lambda>0 .
$$

Proof. By (1.25) and (1.26), for $\hat{F}=\hat{F}_{\varepsilon, x}$ defined as in (4.18) we have

$$
\begin{aligned}
\hat{F}(\lambda) & =\exp \left(-U^{\lambda \varepsilon^{-2}, \varepsilon}(x)\right)-\exp \left(-U^{\infty, \varepsilon}(x)\right) \\
& \geq\left(U^{\infty, \varepsilon}(x)-U^{\lambda \varepsilon^{-2}, \varepsilon}(x)\right) \exp \left(-U^{\infty, \varepsilon}(x)\right) .
\end{aligned}
$$

Let $R_{4.10}=K_{4.3}+R_{4.6}>R_{4.6}$. Then for $\varepsilon \in\left(0, \varepsilon_{0} / R_{4.10}\right)$ we have $|x| / \varepsilon>R_{4.10}$. Use Corollary 4.7(c) with $R=R_{4.10}$ to get

$$
\begin{aligned}
U^{\infty, \varepsilon}(x)-U^{\lambda \varepsilon^{-2}, \varepsilon}(x) & \geq c_{4.6} D^{\lambda}\left(R_{4.10}\right)\left(R_{4.10} /|x|\right)^{p} \varepsilon^{p-2} \\
& \geq c_{4.6} D^{\lambda}\left(R_{4.10}\right) R_{4.10}^{p} \varepsilon_{0}^{-p} \varepsilon^{p-2} .
\end{aligned}
$$

Next, since $|x| / \varepsilon \geq \varepsilon_{0} / \varepsilon>R_{4.10}>K_{4.3}$, we may apply (4.7) to get

$$
\begin{aligned}
\exp \left(-U^{\infty, \varepsilon}(x)\right) & =\exp \left(-\varepsilon^{-2} U^{\infty, 1}(x / \varepsilon)\right) \geq \exp \left(-\varepsilon^{-2} 3(4-d)(|x| / \varepsilon)^{-2}\right) \\
& \geq \exp \left(-9 \varepsilon_{0}^{-2}\right)
\end{aligned}
$$

So the lemma follows from (4.19) and the above inequalities.

Proposition 4.11. There are positive constants $K_{4.11}\left(\varepsilon_{0}\right)$ and $c_{4.11}\left(\varepsilon_{0}\right)$ such that, for all $\varepsilon_{0} \leq|x| \leq \varepsilon_{0}^{-1}$ we have

$$
\mathbb{P}_{\delta_{x}}\left(0<\frac{X_{G_{\varepsilon}}(1)}{\varepsilon^{2}} \leq K_{4.11}\left(\varepsilon_{0}\right)\right) \geq c_{4.11}\left(\varepsilon_{0}\right) \varepsilon^{p-2} \quad \forall 0<\varepsilon<\varepsilon_{0} / R_{4.10}
$$

ProOF. Recall $F=F_{\varepsilon, x}$ and $\hat{F}=\hat{F}_{\varepsilon, x}$ from (4.17) and (4.18), respectively. We have

$$
\hat{F}(\lambda)=\int_{0}^{\infty} e^{-\lambda y} d F(y) .
$$

Let $\lambda=1$ and $K>1$. Use integration by parts and Proposition 4.9 to see that

$$
\begin{aligned}
\hat{F}(1) & =\int_{0}^{\infty} e^{-y} F(y) d y \leq F(K)+\int_{K}^{\infty} e^{-y} F(y) d y \\
& \leq F(K)+\int_{K}^{\infty} e^{-y} c_{4.9}\left(\varepsilon_{0}\right) y^{p-2} \varepsilon^{p-2} d y \\
& \leq F(K)+\frac{1}{2} c_{4.10}\left(\varepsilon_{0}\right) D^{1}\left(R_{4.10}\right) \varepsilon^{p-2},
\end{aligned}
$$

where $K=K\left(\varepsilon_{0}\right)>1$ is large enough. Lemma 4.10, with $\lambda=1$ and $\varepsilon, x$ as in the proposition, implies

$$
F(K) \geq c_{4.10} D^{1}\left(R_{4.10}\right) \varepsilon^{p-2}-\frac{c_{4.10}}{2} D^{1}\left(R_{4.10}\right) \varepsilon^{p-2}=\frac{c_{4.10}}{2} D^{1}\left(R_{4.10}\right) \varepsilon^{p-2} .
$$

Proof of TheOREM 4.1. Pick $\lambda=\lambda\left(\varepsilon_{0}\right) \geq 2(4-d)$ large enough so that

$$
e\left(2^{p} /|x|^{p}\right) D^{\lambda}(2) \leq e 2^{p} \varepsilon_{0}^{-p} D^{\lambda}(2) \leq \frac{1}{2} c_{4.11}\left(\varepsilon_{0}\right) .
$$


So for $K_{1}\left(\varepsilon_{0}\right) \in\left(0,1 / \lambda\left(\varepsilon_{0}\right)\right)$, Proposition 4.8 gives

$$
\mathbb{P}_{\delta_{x}}\left(0<\frac{X_{G_{\varepsilon}}(1)}{\varepsilon^{2}} \leq K_{1}\right) \leq \frac{1}{2} c_{4.11}\left(\varepsilon_{0}\right) \varepsilon^{p-2} .
$$

Let $K_{2}=K_{4.11}$ in Proposition 4.11 to see that for $x, \varepsilon$ as in the Theorem and $R_{4.1}=R_{4.10}$,

$$
\begin{aligned}
\mathbb{P}_{\delta_{x}}\left(K_{1} \leq \frac{X_{G_{\varepsilon}(1)}}{\varepsilon^{2}} \leq K_{2}\right)= & \mathbb{P}_{\delta_{x}}\left(0<\frac{X_{G_{\varepsilon}}(1)}{\varepsilon^{2}} \leq K_{2}\right) \\
& -\mathbb{P}_{\delta_{x}}\left(0<\frac{X_{G_{\varepsilon}}(1)}{\varepsilon^{2}} \leq K_{1}\right) \\
\geq & \frac{1}{2} c_{4.11}\left(\varepsilon_{0}\right) \varepsilon^{p-2} .
\end{aligned}
$$

Use Proposition 2.3(b)(i) with $G=G_{\varepsilon}$ and $D_{1}=G_{\varepsilon / 2}$ to see that for $x, \varepsilon$ as above,

$$
\begin{aligned}
& \mathbb{P}_{\delta_{x}}\left(K_{1} \leq \frac{X_{G_{\varepsilon}}(1)}{\varepsilon^{2}} \leq K_{2}, X_{G_{\varepsilon / 2}}(1)=0\right) \\
& \quad=\mathbb{E}_{\delta_{x}}\left(1\left(K_{1} \leq \frac{X_{G_{\varepsilon}}(1)}{\varepsilon^{2}} \leq K_{2}\right) \mathbb{P}_{X_{G_{\varepsilon}}}\left(X_{G_{\varepsilon / 2}}(1)=0\right)\right) \\
& =\mathbb{E}_{\delta_{x}}\left(1\left(K_{1} \leq \frac{X_{G_{\varepsilon}}(1)}{\varepsilon^{2}} \leq K_{2}\right) \exp \left(-\int U^{\infty, \varepsilon / 2}(y) X_{G_{\varepsilon}}(d y)\right)\right) \\
& =\mathbb{E}_{\delta_{x}}\left(1\left(K_{1} \leq \frac{X_{G_{\varepsilon}}(1)}{\varepsilon^{2}} \leq K_{2}\right) \exp \left(-4 \varepsilon^{-2} U^{\infty, 1}(2) X_{G_{\varepsilon}}(1)\right)\right) \\
& \geq \mathbb{E}_{\delta_{x}}\left(1\left(K_{1} \leq \frac{X_{G_{\varepsilon}}(1)}{\varepsilon^{2}} \leq K_{2}\right) \exp \left(-4 K_{2} U^{\infty, 1}(2)\right)\right) \\
& \geq \frac{1}{2} c_{4.11}\left(\varepsilon_{0}\right) \varepsilon^{p-2} \exp \left(-4 K_{2} U^{\infty, 1}(2)\right),
\end{aligned}
$$

the second equality by (1.26) with $X_{0}=X_{G_{\varepsilon}}$ and the last inequality by (4.20). So the theorem follows.

5. Preliminaries for the lower bound on the dimension. In this section, we will show that the lower bound on the local dimension of $\partial \mathcal{R}$ holds with positive probability (see Proposition 5.3). An extended version of this result, which is crucial for the later proof of Proposition 1.7, is given in Lemma 5.4. The next result is important for implementing our program: it plays a role analogous to that of Proposition 6.1 in [14].

PROPOSITION 5.1. There is a $\lambda>0$ such that for all $\varepsilon_{0}>0$, there is some $c_{5.1}\left(\varepsilon_{0}\right)>0$ so that for all $\left|x_{i}\right| \geq \varepsilon_{0}$ and $\varepsilon \in\left(0, \varepsilon_{0}\right)$,

$$
\mathbb{E}_{\delta_{0}}\left(\prod_{i=1}^{2} \lambda \frac{X_{G_{\varepsilon}}^{x_{i}}(1)}{\varepsilon^{2}} \exp \left(-\lambda \frac{X_{G_{\varepsilon}}^{x_{i}}(1)}{\varepsilon^{2}}\right)\right) \leq c_{5.1}\left(1+\left|x_{1}-x_{2}\right|^{2-p}\right) \varepsilon^{2(p-2)} .
$$

Given the results in Section 4, the proof then follows from that of Proposition 6.1 in [14], but as there are some differences, it is included in Supplementary Material [9]. The reader should note that the role of $\lambda$ in [14] is now played by $\lambda \varepsilon^{-2}$, where $\lambda$ is chosen to be a fixed large constant.

Recall that we are in the case $d=2$ or 3 . Let $\beta>0$ and $g_{\beta}(r)=r^{-\beta}$. For a finite measure $\mu$ on $\mathbb{R}^{d}$ and Borel subset $A$ of $\mathbb{R}^{d}$, let

$$
\langle\mu\rangle_{g_{\beta}}=\iint g_{\beta}(|x-y|) d \mu(x) d \mu(y),
$$


and

$$
I\left(g_{\beta}\right)(A)=\inf \left\{\langle\mu\rangle_{g_{\beta}}: \mu \text { is a probability supported by } A\right\} .
$$

The $g_{\beta}$-capacity of $A$ is $C\left(g_{\beta}\right)(A)=\left(I\left(g_{\beta}\right)(A)\right)^{-1}$. Set

$$
\beta=p-2= \begin{cases}2 \sqrt{2}-2 & \text { if } d=2, \\ (\sqrt{17}-3) / 2 & \text { if } d=3,\end{cases}
$$

and note $\beta \in(1 / 2,1)$. Now we may use Theorem 4.1 and Proposition 5.1 to get the following theorem. Although similar to the omitted proof of Theorem 6.2 in [14], there are some important adjustments, and so this time the argument is included.

THEOREM 5.2. Assume $d=2$ or 3 . For every $\varepsilon_{0} \in(0,1)$, there is some $c_{5.2}\left(\varepsilon_{0}\right)>0$ such that for any Borel set, $A$, of $\left\{x \in \mathbb{R}^{d}: \varepsilon_{0} \leq|x| \leq \varepsilon_{0}^{-1}\right\}$,

$$
\mathbb{P}_{\delta_{0}}(\partial \mathcal{R} \cap A \neq \varnothing) \geq c_{5.2}\left(\varepsilon_{0}\right) C\left(g_{\beta}\right)(A) .
$$

In particular, for any Borel subset $A$ of $\mathbb{R}^{d}, C\left(g_{\beta}\right)(A)>0$ implies that $\mathbb{P}_{\delta_{0}}(\partial \mathcal{R} \cap A \neq \varnothing)>0$.

PROOF. Fix $\varepsilon_{0} \in(0,1)$. Let $\Gamma \subset\left\{x \in \mathbb{R}^{d}: \varepsilon_{0} \leq|x| \leq \varepsilon_{0}^{-1}\right\}$ be compact and such that (without loss of generality) $C(\Gamma)=C\left(g_{\beta}\right)(\Gamma)>0$. If $I(\Gamma)=I\left(g_{\beta}\right)(\Gamma)$, we may choose $\left\{x_{i}^{N}: 1 \leq i \leq N\right\} \subset \Gamma$ so that (suppressing the superscript $N$ ) as $N \rightarrow \infty$ (see [19]),

$$
I_{N} \equiv \frac{1}{N(N-1)} \sum_{i} \sum_{j \neq i}\left|x_{i}-x_{j}\right|^{-\beta} \rightarrow I(\Gamma)=1 / C(\Gamma) .
$$

Let

$$
\hat{\Gamma}=\left\{x_{i}^{N}: 1 \leq i \leq N, N \geq 1\right\} \subset \Gamma .
$$

For any fixed $\varepsilon \in\left(0, \varepsilon_{0}\right)$, we define $\partial \mathcal{R}(\varepsilon, \Gamma) \subset \Gamma$ to be

$$
\partial \mathcal{R}(\varepsilon, \Gamma):=\left\{x \in \hat{\Gamma}: K_{1} \varepsilon^{2} \leq X_{G_{\varepsilon}^{x}}(1) \leq K_{2} \varepsilon^{2}, X_{G_{\varepsilon / 2}^{x}}(1)=0\right\},
$$

where $0<K_{1}\left(\varepsilon_{0}\right)<K_{2}\left(\varepsilon_{0}\right)<\infty$ are as in Theorem 4.1. Note the exit measure $X_{G_{\varepsilon}^{x}}$ is constructed up to a null set for each $(x, \varepsilon)$, so the reader should note that we will only be considering at most countably many values of $(x, \varepsilon)$ at a time as is the case in the above definition for a fixed $\varepsilon$. Let $\lambda>0$ be as in Proposition 5.1. There is some $\delta=\delta\left(\lambda, \varepsilon_{0}\right) \in$ $\left(0, e^{-1}\right)$ such that

$$
\lambda \frac{X_{G_{\varepsilon}^{x}}(1)}{\varepsilon^{2}} \exp \left(-\lambda \frac{X_{G_{\varepsilon}^{x}}(1)}{\varepsilon^{2}}\right) \geq \delta \quad \forall x \in \partial \mathcal{R}(\varepsilon, \Gamma) .
$$

Therefore, by translation invariance, inclusion-exclusion, Theorem 4.1, (5.2) and Proposition 5.1 , for $\varepsilon \in\left(0, \varepsilon_{0} / R_{4.1}\right)$,

$$
\begin{aligned}
& \mathbb{P}_{\delta_{0}}(\partial \mathcal{R}(\varepsilon, \Gamma) \neq \varnothing) \\
& \quad \geq \mathbb{P}_{\delta_{0}}\left(\bigcup_{i=1}^{N}\left\{x_{i} \in \partial \mathcal{R}(\varepsilon, \Gamma)\right\}\right) \\
& \quad \geq \sum_{i=1}^{N} \mathbb{P}_{\delta_{0}}\left(x_{i} \in \partial \mathcal{R}(\varepsilon, \Gamma)\right)-\sum_{i} \sum_{j \neq i} \mathbb{P}_{\delta_{0}}\left(x_{i}, x_{j} \in \partial \mathcal{R}(\varepsilon, \Gamma)\right) \\
& \quad \geq N c_{4.1}\left(\varepsilon_{0}\right) \varepsilon^{p-2}-\sum_{i} \sum_{j \neq i} \delta^{-2} \mathbb{E}_{\delta_{0}}\left(\prod_{k=i, j} \lambda \frac{X_{G_{\varepsilon}^{x_{k}}}(1)}{\varepsilon^{2}} \exp \left(-\lambda \frac{X_{G_{\varepsilon}^{x_{k}}}(1)}{\varepsilon^{2}}\right)\right)
\end{aligned}
$$




$$
\begin{aligned}
& \geq N c_{4.1} \varepsilon^{p-2}-c_{5.1} \delta^{-2} \varepsilon^{2(p-2)} \sum_{i} \sum_{j \neq i}\left(1+\left|x_{i}-x_{j}\right|^{2-p}\right) \\
& \geq c_{4.1} N \varepsilon^{p-2}-C\left(\varepsilon_{0}\right)\left(N \varepsilon^{p-2}\right)^{2} I_{N} .
\end{aligned}
$$

Now choose $\varepsilon_{N} \rightarrow 0$ such that $N \varepsilon_{N}^{p-2}=c_{4.1} /\left(2 C\left(\varepsilon_{0}\right) I_{N}\right)$. Therefore, for some $c\left(\varepsilon_{0}\right)>0$,

$$
\mathbb{P}_{\delta_{0}}\left(\partial \mathcal{R}\left(\varepsilon_{N}, \Gamma\right) \neq \varnothing\right) \geq \frac{c_{4.1}}{2 C\left(\varepsilon_{0}\right) I_{N}} \frac{c_{4.1}}{2} \rightarrow c\left(\varepsilon_{0}\right) C(\Gamma) \quad \text { as } N \rightarrow \infty .
$$

This implies

$$
\mathbb{P}_{\delta_{0}}\left(\partial \mathcal{R}\left(\varepsilon_{N}, \Gamma\right) \neq \varnothing, \text { infinitely often }\right) \geq c\left(\varepsilon_{0}\right) C(\Gamma) .
$$

Assume now that

$$
\omega \in\left\{\partial \mathcal{R}\left(\varepsilon_{N}, \Gamma\right) \neq \varnothing, \text { infinitely often }\right\} .
$$

So we may choose $\left\{z_{N}\right\} \subset \hat{\Gamma} \subset \Gamma$ such that $z_{N} \in \partial \mathcal{R}\left(\varepsilon_{N}, \Gamma\right)$, where we have suppressed the further subsequence of $\varepsilon_{N}$ in our notation. The definition of $\partial \mathcal{R}\left(\varepsilon_{N}, \Gamma\right)$ gives $X_{G_{\varepsilon_{N}}^{z_{N}}}(1)>$ 0 and $X_{G_{\varepsilon_{N} / 2}^{z_{N}}}(1)=0$. By Proposition 2.3(b)(ii) and translation invariance, $X_{G_{\varepsilon_{N} / 2}^{z_{N}}}(1)=0$ implies

$$
\mathcal{R} \cap B\left(z_{N}, \varepsilon_{N} / 3\right)=\varnothing
$$

By (2.3), $X_{G_{\varepsilon_{N}}^{z_{N}}}(1)>0$ implies

$\mathcal{R} \cap \partial G_{\varepsilon_{N}}^{z_{N}}$ is nonempty.

Combining (5.3) and (5.4) with an elementary argument in point set topology, we can choose $y_{N} \in \partial \mathcal{R}$ such that $\varepsilon_{N} / 4 \leq\left|y_{N}-z_{N}\right| \leq \varepsilon_{N}$. The compactness of $\Gamma$ implies there is some $x \in \Gamma$ such that $z_{N_{k}} \rightarrow x$ as $N_{k} \rightarrow \infty$. Therefore, $y_{N_{k}} \rightarrow x$ and $x \in \partial \mathcal{R}$ since $\partial \mathcal{R}$ is closed, which gives $x \in \Gamma \cap \partial \mathcal{R} \neq \varnothing$, and so the proof is complete for $A=\Gamma$ compact. Use the inner regularity of capacity to extend the inequality to any Borel subset of $\left\{x \in \mathbb{R}^{d}: \varepsilon_{0} \leq\right.$ $\left.|x| \leq \varepsilon_{0}^{-1}\right\}$, and then conclude for any Borel subset $A$ of $\mathbb{R}^{d}$ that $C\left(g_{\beta}\right)(A)>0$ implies that $\mathbb{P}_{\delta_{0}}(\partial \mathcal{R} \cap A \neq \varnothing)>0$.

Proposition 5.3. For each nonempty open set $B$ in $\mathbb{R}^{d}$ :

(a) $\mathbb{P}_{\delta_{0}}\left(\operatorname{dim}(\partial \mathcal{R} \cap B) \geq d_{f}\right)>0$.

(b) $\mathbb{N}_{0}\left(\operatorname{dim}(\partial \mathcal{R} \cap B) \geq d_{f}\right):=p_{5.3}(B)>0$.

PROOF. (a) is derived from Theorem 5.2 by taking $A$ to be the range of an appropriate independent Lévy process, exactly as in the proof of Proposition 6.5 of [14]. (b) then follows easily from (a) by making trivial changes to the cluster decomposition proof of Corollary 6.6 in [14].

To help upgrade the lower bound in part (a) of the above to probability one, we need to extend (a) to more general initial conditions through a scaling argument.

LEMMA 5.4. There is a constant $q_{5.4}>0$ so that if $X_{0}^{\prime} \in M_{F}\left(\mathbb{R}^{d}\right)$ is supported on $\{|x|=$ $r$ \} and $\delta=X_{0}^{\prime}(1)$ satisfies $0<\delta \leq r^{2}$, then

$$
\mathbb{P}_{X_{0}^{\prime}}\left(\operatorname{dim}\left(\partial \mathcal{R} \cap B\left(0, r-\frac{\sqrt{\delta}}{2}\right)\right) \geq d_{f}\right) \geq q_{5.4}
$$


Proof. Define $X_{0}^{(\delta)}(A)=\delta^{-1} X_{0}^{\prime}(\sqrt{\delta} A)$, so that the support of $X_{0}^{(\delta)}$ is on $\{|x|=r / \sqrt{\delta}\}$ and has total mass one. By scaling properties of SBM (see, e.g., Ex. II.5.5 in [15]) and scale invariance of Hausdorff dimension, we may conclude that

$$
\begin{aligned}
& \mathbb{P}_{X_{0}^{\prime}}\left(\operatorname{dim}\left(\partial \mathcal{R} \cap B\left(0, r-\frac{\sqrt{\delta}}{2}\right)\right) \geq d_{f}\right) \\
& \quad=\mathbb{P}_{X_{0}^{(\delta)}}\left(\operatorname{dim}\left(\partial \mathcal{R} \cap B\left(0, \frac{r}{\sqrt{\delta}}-\frac{1}{2}\right)\right) \geq d_{f}\right) .
\end{aligned}
$$

Now work in our standard set-up for SBM with initial law $X_{0}^{(\delta)}$ so that (by (2.1)), $X_{t}=$ $\sum_{j \in J} X_{t}\left(W_{j}\right)=\int X_{t}(W) \Xi(d W)$ for all $t>0$, where $\Xi$ is a Poisson point process with intensity $\mathbb{N}_{X_{0}^{(\delta)}}$. For $r \geq \sqrt{\delta}$, define

$$
\begin{aligned}
\tau_{\rho}\left(W_{j}\right) & =\inf \left\{t \geq 0:\left|\hat{W}_{j}(t)\right| \leq \rho\right\}, \\
U_{\rho}\left(W_{j}\right) & =\inf \left\{t \geq 0:\left|\hat{W}_{j}(t)-\hat{W}_{j}(0)\right| \geq \rho\right\}, \quad \text { and } \\
N_{1} & =\sum_{j \in J} 1\left(\tau_{(r / \sqrt{\delta})-(1 / 2)}\left(W_{j}\right)<\infty\right):=\#\left(I_{r, \delta}\right) .
\end{aligned}
$$

Here as usual $\inf \varnothing=\infty$. Then $N_{1}$ is Poisson with mean

$$
\begin{aligned}
m_{r, \delta} & :=\mathbb{N}_{X_{0}^{(\delta)}}\left(\tau_{(r / \sqrt{\delta})-(1 / 2)}<\infty\right) \leq \mathbb{N}_{X_{0}^{(\delta)}}\left(U_{1 / 2}(W)<\infty\right) \\
& =\mathbb{N}_{0}\left(U_{1 / 2}(W)<\infty\right):=\bar{m}<\infty,
\end{aligned}
$$

where $X_{0}^{(\delta)}(1)=1$ and translation invariance are used in the equality, and the finiteness of $\bar{m}$ follows from Theorem 1 of [10].

Let $\mathcal{R}\left(W_{j}\right)=\left\{\hat{W}_{j}(t): t \leq \sigma\left(W_{j}\right)\right\}$ (recall (2.2)) be the range of the $j$ th excursion, so that

$$
\mathcal{R} \cap B\left(0, \frac{r}{\sqrt{\delta}}-\frac{1}{2}\right)=\bigcup_{j \in J, \tau_{(r / \sqrt{\delta})-(1 / 2)}\left(W_{j}\right)<\infty}\left(\mathcal{R}\left(W_{j}\right) \cap B\left(0, \frac{r}{\sqrt{\delta}}-\frac{1}{2}\right)\right) .
$$

We may assume (by additional randomization) that conditional on $I_{r, \delta}$, we have $\left\{W_{j}: j \in\right.$ $\left.I_{r, \delta}\right\}$ are i.i.d. with law $\mathbb{N}_{X_{0}^{(\delta)}}\left(W \in \cdot \mid \tau_{(r / \sqrt{\delta})-(1 / 2)}<\infty\right)$. Therefore, the right-hand side of (5.5) is at least

$$
\begin{gathered}
\mathbb{P}_{X_{0}^{(\delta)}}\left(N_{1}=1\right) \mathbb{N}_{X_{0}^{(\delta)}}\left(\operatorname{dim}\left(\partial \mathcal{R} \cap B\left(0, \frac{r}{\sqrt{\delta}}-\frac{1}{2}\right)\right) \geq d_{f} \mid \tau_{\frac{r}{\sqrt{\delta}}-\frac{1}{2}}<\infty\right) \\
=\frac{m_{r, \delta} e^{-m_{r, \delta}}}{m_{r, \delta}} \mathbb{N}_{x_{0}}\left(\operatorname{dim}\left(\partial \mathcal{R} \cap B\left(0, \frac{r}{\sqrt{\delta}}-\frac{1}{2}\right)\right) \geq d_{f}\right),
\end{gathered}
$$

where $x_{0}=\left(\frac{r}{\sqrt{\delta}}\right) e_{1}$ and $e_{1}$ is the first unit basis vector. We also have used the fact that spherical symmetry shows we could have taken any $x_{0}$ on the sphere of radius $r / \sqrt{\delta}$. Now again use translation invariance and spherical symmetry to see that the right-hand side of (5.7) equals

$$
\begin{aligned}
& e^{-m_{r, \delta}} \mathbb{N}_{0}\left(\operatorname{dim}\left(\partial \mathcal{R} \cap B\left(x_{0},\left|x_{0}\right|-\frac{1}{2}\right)\right) \geq d_{f}\right) \\
& \geq e^{-m_{r, \delta} \mathbb{N}_{0}\left(\operatorname{dim}\left(\partial \mathcal{R} \cap B\left(e_{1}, 1 / 2\right)\right) \geq d_{f}\right)} \\
& \geq e^{-\bar{m}} \mathbb{N}_{0}\left(\operatorname{dim}\left(\partial \mathcal{R} \cap B\left(e_{1}, 1 / 2\right)\right) \geq d_{f}\right) \\
& \geq e^{-\bar{m}} p_{5.3}\left(B\left(e_{1}, 1 / 2\right)\right)>0 \text {, }
\end{aligned}
$$


where the first inequality follows by $B\left(e_{1}, 1 / 2\right) \subset B\left(x_{0},\left|x_{0}\right|-1 / 2\right)$, the second inequality uses (5.6) and the first inequality in the last line holds by Proposition 5.3(b). We have shown that the right-hand side of (5.8) is a lower bound for (5.5), and so have proved the lemma with $q_{5.4}=e^{-\bar{m}} p_{5.3}\left(B\left(e_{1}, 1 / 2\right)\right)$.

6. Exit measures and continuous state branching processes. Throughout this section, we assume that $r_{0}>0$ and the initial state $X_{0}$ satisfy

$$
B_{2 r_{0}} \subset \operatorname{Supp}\left(X_{0}\right)^{c} \text {. }
$$

To complete the proofs of Propositions 1.6, 1.7, we need to establish some properties of the total exit measure process $X_{G_{r_{0}-r}}(1), 0 \leq r<r_{0}$. We will show below in Proposition 6.2 that, for any $r_{0}>0$, the "time changed" process $Z_{t}=X_{G_{r_{0}} e^{-t}}(1) /\left(r_{0} e^{-t}\right)^{2}, t \geq 0$, is a time homogeneous continuous state branching process (CSBP), and thus has no negative jumps.

A nonnegative function $\lambda \mapsto u(\lambda)$ on $[0, \infty)$ is completely concave iff for every $y>0$, $\lambda \rightarrow \exp (-y u(\lambda))$ is the Laplace transform of a probability measure on the half-line. (See (4.1) in [17] for a Lévy-Khintchine representation of such functions). We recall the definition of a continuous state branching process from Section 4 of [17].

Definition. A (finite) continuous state branching process (CSBP) $Z$ is a timehomogeneous $[0, \infty)$-valued Markov process with no fixed time discontinuities (if $t_{n} \rightarrow t$, then $Z\left(t_{n}\right) \rightarrow Z(t)$ a.s.), and such that there is a family of completely concave functions $\{u(s, \cdot): s>0\}$ satisfying

$$
\begin{aligned}
& E\left(\exp \left(-\lambda Z\left(t_{2}\right)\right) \mid Z(s), s \leq t_{1}\right) \\
& \quad=\exp \left(-Z\left(t_{1}\right) u\left(t_{2}-t_{1}, \lambda\right)\right) \quad \text { a.s. } \forall t_{2}>t_{1} \geq 0, \lambda \geq 0 .
\end{aligned}
$$

The associated family $\{u(t, \cdot): t>0\}$ is the log-Laplace transform of $Z$.

Recall that $U^{\lambda, R}(x)=U^{\lambda, R}(|x|)$ is the unique continuous map on $\{|x| \geq R\}$ which is $C^{2}$ on $G_{R}$ and satisfies

$$
\Delta U=U^{2} \quad \text { on } G_{R} \quad \text { and } \quad U=\lambda \quad \text { on } \partial G_{R} .
$$

A simple application of the comparison principle (e.g., Chapter V, Lemma 7 of [12]), using the last part of (1.27), gives

$$
U^{\lambda, R}(x) \leq \lambda \quad \forall|x| \geq R
$$

Define

$$
u(t, \lambda)=e^{2 t} U^{\lambda, 1}\left(e^{t}\right) \quad \text { for } t \geq 0
$$

Notation. For $0 \leq r<r_{0}$, we define $Y(r)(\cdot)=X_{G_{r_{0}-r}}(\cdot)$ and let $\mathcal{E}_{r}=\mathcal{E}_{G_{r_{0}-r}} \vee$ $\sigma\left(\left\{\mathbb{N}_{X_{0}}-\right.\right.$ null sets $\left.\}\right)$. For $t \geq 0$ set

$$
Z(t)=X_{G_{r_{0} e^{-t}}}(1) \frac{e^{2 t}}{r_{0}^{2}}=Y\left(r_{0}\left(1-e^{-t}\right)\right)(1) e^{2 t} r_{0}^{-2} ; \quad \mathcal{G}_{t}=\mathcal{E}_{r_{0}\left(1-e^{-t}\right)}=\mathcal{E}_{G_{r_{0} e^{-t}}} .
$$

It is not hard to show that $\mathcal{E}_{r}$ is nondecreasing in $r$ (the corresponding result for halfspaces is noted prior to (7.2) of [14] and the observation made there applies to balls as well.) By Proposition 2.3 of [11], $Y$ is $\left(\mathcal{E}_{r}\right)$-adapted and $Z$ is $\left(\mathcal{G}_{t}\right)$-adapted. Let $\mathcal{E}_{r}^{+}=\mathcal{E}_{r+}$ denote the associated right-continuous filtration. In addition to $\mathbb{N}_{X_{0}}$, we will also work under the 
probability $Q_{X_{0}}(\cdot)=\mathbb{N}_{X_{0}}\left(\cdot \mid Y_{0}(1)>0\right)$, where (6.1) ensures that $\mathbb{N}_{X_{0}}\left(Y_{0}(1)>0\right)<\infty$. Note that

$$
\text { for any r.v. } Z \geq 0 \text {, and any } r \geq 0, \quad Q_{X_{0}}\left(Z \mid \mathcal{E}_{r}\right)=\mathbb{N}_{X_{0}}\left(Z \mid \mathcal{E}_{r}\right) \quad Q_{X_{0}} \text {-a.s. }
$$

because $\left\{Y_{0}(1)>0\right\} \in \mathcal{E}_{0}$. When conditioning on $\mathcal{E}_{r}$ under $Q_{X_{0}}$, we are adding the slightly larger class of $Q_{X_{0}}$-null sets to $\mathcal{E}_{r}$, but will not record this distinction in our notation. Below we will apply the definition of (CSBP) under the $\sigma$-finite measure $\mathbb{N}_{X_{0}}$ as well as $Q_{X_{0}}$. We write $Q_{x_{0}}$ for $Q_{\delta_{x_{0}}}$ as usual.

LEMMA 6.1. (a) If $0 \leq t_{1}<t_{2}$ and $\lambda \geq 0$, then:

(i) $Q_{X_{0}}\left(e^{-\lambda Z_{t_{2}}} \mid \mathcal{G}_{t_{1}}\right)=\mathbb{N}_{X_{0}}\left(e^{-\lambda Z_{t_{2}}} \mid \mathcal{G}_{t_{1}}\right)=\exp \left(-Z_{t_{1}} u\left(t_{2}-t_{1}, \lambda\right)\right)$.

(ii)

$$
\begin{aligned}
Q_{X_{0}}\left(\left(e^{-\lambda Z_{t_{2}}}-e^{-\lambda Z_{t_{1}}}\right)^{2}\right)= & Q_{X_{0}}\left(\exp \left(-Z_{t_{1}} u\left(t_{2}-t_{1}, 2 \lambda\right)\right)\right. \\
& -2 \exp \left(-\lambda Z_{t_{1}}-Z_{t_{1}} u\left(t_{2}-t_{1}, \lambda\right)\right) \\
& \left.+\exp \left(-2 \lambda Z_{t_{1}}\right)\right),
\end{aligned}
$$

and similarly for $\mathbb{N}_{X_{0}}$.

(b) For all $t>0, \lambda \mapsto u(t, \lambda)$ is completely concave.

(c) $\left(Z_{t}, t \geq 0\right)$ is a (time-homogeneous) $\left(\mathcal{G}_{t}\right)$-Markov process under $Q_{X_{0}}$ or $\mathbb{N}_{X_{0}}$.

PROOF. (a) (6.6) shows that for $\lambda \geq 0$, the left-hand side of (i) equals the middle expression, which by Proposition 2.2(a)(ii) and then (1.25) equals

$$
\begin{aligned}
& \mathbb{E}_{X_{G_{r_{0}} e^{-t_{1}}}}\left(\exp \left(-\lambda e^{2 t_{2}} r_{0}^{-2} X_{G_{r_{0} e^{-t_{2}}}}(1)\right)\right) \\
& =\exp \left(-\int U^{\lambda e^{2 t_{2} r_{0}^{-2}}, r_{0} e^{-t_{2}}}(x) X_{G_{r_{0} e^{-t_{1}}}}(d x)\right) \\
& =\exp \left(-U^{\lambda e^{2 t_{2}} r_{0}^{-2}, r_{0} e^{-t_{2}}}\left(r_{0} e^{-t_{1}}\right) X_{G_{r_{0} e^{-t_{1}}}}(1)\right) \\
& =\exp \left(-u\left(t_{2}-t_{1}, \lambda\right) Z_{t_{1}}\right),
\end{aligned}
$$

where scaling (i.e., (1.24)) is used in the last line. This gives (i). It is then easy to derive (ii) by expanding out the square, conditioning on $\mathcal{G}_{t_{1}}$ and finally using (i).

(b) Let $y_{0}>0$ and $t>0$. Let $m_{r}$ be the uniform distribution on $\{|x|=r\}$ and set $W=$ $e^{2 t} r_{0}^{-2} X_{G_{r_{0} e^{-t}}}(1)$. Apply (1.25) and then scaling ((1.24)) to see that for all $\lambda \geq 0$,

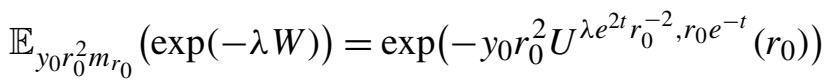

$$
\begin{aligned}
& =\exp \left(-y_{0} r_{0}^{2} r_{0}^{-2} e^{2 t} U^{\lambda, 1}\left(e^{t}\right)\right) \\
& =\exp \left(-y_{0} u(t, \lambda)\right) \text {. }
\end{aligned}
$$

(c) This is immediate from (a)(i), (b) (to define the family of laws $\left\{P_{x}: x \geq 0\right\}$ ), and a monotone class argument.

Proposition 1.8 in the Introduction follows from part (c) of our next result. 
PRoposition 6.2. (a) $Y$ is an inhomogeneous $\left(\mathcal{E}_{r}\right)$-Markov process under $\mathbb{N}_{X_{0}}$ or $Q_{X_{0}}$. That is, for any $\psi: M_{F}\left(\mathbb{R}^{d}\right) \rightarrow[0, \infty)$ Borel measurable and for any $0 \leq r_{1}<r_{2}$, we have

$$
Q_{X_{0}}\left(\psi\left(Y\left(r_{2}\right)\right) \mid \mathcal{E}_{r_{1}}\right)=\mathbb{N}_{X_{0}}\left(\psi\left(Y\left(r_{2}\right)\right) \mid \mathcal{E}_{r_{1}}\right)=\mathbb{E}_{Y\left(r_{1}\right)}\left(\psi\left(Y\left(r_{2}\right)\right)\right) \quad \text { a.s. }
$$

(b) If $0 \leq r_{1}<r_{2}<r_{0}$, then the total mass, $Y_{r}(1)$, of $Y_{r}$ satisfies

$$
\mathbb{N}_{X_{0}}\left(Y_{r_{2}}(1) \mid \mathcal{E}_{r_{1}}\right)= \begin{cases}Y_{r_{1}}(1) & \text { if } d=2, \\ \frac{r_{0}-r_{2}}{r_{0}-r_{1}} Y_{r_{1}}(1) & \text { if } d=3 .\end{cases}
$$

Under $\mathbb{N}_{X_{0}}$ or $Q_{X_{0}}, Y_{r}(1)$ has a cadlag version on $\left[0, r_{0}\right)$ which is an $\left(\mathcal{E}_{r}^{+}\right)$-supermartingale $\left(\right.$ an $\left(\mathcal{E}_{r}^{+}\right)$-martingale if $d=2$ ), satisfies $(6.8)$ with $\mathcal{E}_{r_{1}}^{+}$in place of $\mathcal{E}_{r_{1}}$, and has only nonnegative jumps a.e.

(c) Under $\mathbb{N}_{X_{0}}$ or $Q_{X_{0}},(Z(t), t \geq 0)$ has a cadlag version with only nonnegative jumps which is a CSBP with log Laplace transform given by $\{u(t, \cdot): t>0\}$ in (6.5).

ProOF. (a) This is immediate from Proposition 2.2(a)(ii) and (6.6).

$(\mathrm{b}, \mathrm{c})$ Let $B$ denote a $d$-dimensional Brownian motion starting at $x$ under $P_{x}^{B}$ and

$$
\tau_{r}=\inf \left\{t \geq 0:\left|B_{t}\right| \leq r\right\} \quad(\inf \varnothing=\infty) .
$$

Recalling (6.1), Proposition 3 in Chapter $\mathrm{V}$ of [12] shows that for $0 \leq r<r_{0}$,

$$
\begin{aligned}
\mathbb{N}_{X_{0}}\left(Y_{r}(1)\right) & =\int P_{x}^{B}\left(\tau_{r_{0}-r}<\infty\right) d X_{0}(x) \\
& = \begin{cases}X_{0}(1) & \text { if } d=2, \\
\int \frac{r_{0}-r}{|x|} d X_{0}(x) & \text { if } d=3 .\end{cases}
\end{aligned}
$$

Return now to the probability $\mathbb{P}_{X_{0}}$, and use (2.4) and the above to see that

$$
\mathbb{E}_{X_{0}}\left(Y_{r}(1)\right)=\mathbb{N}_{X_{0}}\left(Y_{r}(1)\right)= \begin{cases}X_{0}(1) & \text { if } d=2 \\ \int \frac{r_{0}-r}{|x|} d X_{0}(x) & \text { if } d=3\end{cases}
$$

Although we have assumed $B_{2 r_{0}} \subset \operatorname{Supp}\left(X_{0}\right)^{c}$, both (6.9) and (6.10) will apply if $\operatorname{Supp}\left(X_{0}\right) \subset$ $G_{r_{0}-r}$. This allows us to apply (6.10), with $X_{0}=Y_{r_{1}}$ and $r=r_{2}$, and (a) to derive (6.8).

Turning to the second part of (b) and (c), we first work with $Z$. Let $t_{n} \uparrow t>0\left(t_{n}<t\right)$ and set $r_{n}=r_{0}\left(1-e^{-t_{n}}\right) \uparrow r_{0}\left(1-e^{-t}\right)=r \in\left(0, r_{0}\right)$. By (6.8) and supermartingale convergence, $\left\{Y_{r_{n}}\right\}$ converges $\mathbb{N}_{X_{0}}$-a.e. to a limit we denote by $Y_{r-}$ (1) for now. (The $\sigma$-finiteness of $\mathbb{N}_{X_{0}}$ is not an issue here, but the reader who prefers probabilities may work with $Q_{X_{0}}$ and note that on the complementary set, $\left\{Y_{0}(1)=0\right\}, Y_{r_{n}}(1)=0 \mathbb{N}_{X_{0}}$-a.e. by (6.8) with $r_{1}=0$ there. Henceforth we will not make such arguments.) It follows that

$$
Z_{t_{n}} \rightarrow e^{2 t} r_{0}^{-2} Y_{r-}(1):=Z_{t-} \quad \mathbb{N}_{X_{0}} \text {-a.e. }
$$

By (6.7),

$$
\begin{aligned}
& Q_{X_{0}}\left(\left(e^{-\lambda Z_{t}}-e^{-\lambda Z_{t_{n}}}\right)^{2}\right) \\
& =Q_{X_{0}}\left(\exp \left(-Z_{t_{n}} u\left(t-t_{n}, 2 \lambda\right)\right)-2 \exp \left(-\left(\lambda+u\left(t-t_{n}, \lambda\right)\right) Z_{t_{n}}\right)\right. \\
& \left.\quad+\exp \left(-2 \lambda Z_{t_{n}}\right)\right) \\
& \quad \rightarrow Q_{X_{0}}\left(\exp \left(-2 \lambda Z_{t-}\right)-2 \exp \left(-2 \lambda Z_{t-}\right)+\exp \left(-2 \lambda Z_{t-}\right)\right) \quad \text { as } n \rightarrow \infty \\
& =0,
\end{aligned}
$$


where dominated convergence is used in the above convergence. This and (6.11) show that $Z_{t_{n}} \rightarrow Z_{t} Q_{X_{0}}$-a.s. The fact, noted above, that $Y_{0}(1)=0$ implies $Z_{t_{n}}=Z_{t}=0 \mathbb{N}_{X_{0}}$-a.e. allows us to upgrade this to

$$
Z_{t_{n}} \rightarrow Z_{t} \quad \mathbb{N}_{X_{0}} \text {-a.e. if } t_{n} \uparrow t>0 .
$$

A simpler argument, now using reverse supermartingale convergence, shows that

$$
Z_{t_{n}} \rightarrow Z_{t} \quad \mathbb{N}_{X_{0}} \text {-a.e. if } t_{n} \downarrow t \geq 0 .
$$

(6.12) and (6.13) imply $Y_{r}(1)$ is continuous in measure on $\left[0, r_{0}\right)$. Therefore, by (6.8) there is a cadlag version of $\left(Y_{r}(1), r \in\left[0, r_{0}\right)\right)$ under $\mathbb{N}_{X_{0}}$ (we do not change the notation) which is an $\left(\mathcal{E}_{r}^{+}\right)$-supermartingale (martingale if $d=2$ ) satisfying (6.8) with $\mathcal{E}_{r_{1}}^{+}$in place of $\mathcal{E}_{r_{1}}$. This gives a cadlag version of $Z$ which satisfies the $\left(\mathcal{G}_{t+}\right)$ version of Lemma 6.1(a)(i), and so is $\left(\mathcal{G}_{t+}\right)$-Markov under $\mathbb{N}_{X_{0}}$ or $Q_{X_{0}}$, just as for Lemma 6.1(c). Clearly, (6.12) and (6.13) imply that $Z_{t-}=Z_{t} \mathbb{N}_{X_{0}}$-a.e., and so $Z$ has no fixed time discontinuities. It follows from the above and Lemma 6.1(b) that $\left(Z_{t}, t \geq 0\right)$ is a (CSBP) with $\log$ Laplace transform $\{u(t, \cdot): t>0\}$ under $\mathbb{N}_{X_{0}}$ or $Q_{X_{0}}$. A theorem of Lamperti (see. e.g., page 1044 of [17]) shows that $\left(Z_{t}, t \geq\right.$ $0)$ has only nonnegative jumps a.e. and so the same applies to $\left(Y_{r}(1), r \in\left[0, r_{0}\right]\right)$.

REMARK 6.3. Although in this work we only use the above results, we briefly discuss the processes $Z$. and $Y$.(1) in the general context of CSBPs. By Proposition 6.2(c) above and Theorem 4 of [17], there is a Lévy measure $\tilde{\pi}$ on $[0, \infty)$ satisfying $\int \ell^{2} \wedge 1 d \tilde{\pi}(\ell)<\infty$ and constants $\tilde{a} \in \mathbb{R}, b \geq 0$, such that if

$$
\Psi(u)=\tilde{a} u-b u^{2}+\int_{0}^{\infty}\left(1-e^{-u \ell}-u \ell e^{-\ell}\right) d \tilde{\pi}(\ell), \quad u \geq 0,
$$

then $t \mapsto u(t, \lambda)$ is the unique solution of

$$
\frac{d u(t, \lambda)}{d t}=\Psi(u(t, \lambda)), \quad u(0, \lambda)=\lambda .
$$

$Z$ is often called a $\Psi$-CSBP. (6.14) implies $\Psi$ is concave on $[0, \infty)$ and differentiable on $(0, \infty)$. If

$$
\lambda_{d}=2(4-d)
$$

then a short calculation using (6.5) and (6.3) gives (primes denote derivatives with respect to $t$ )

$$
u^{\prime \prime}(t, \lambda)=(6-d) u^{\prime}+u\left(u-\lambda_{d}\right), \quad t \geq 0 .
$$

Differentiating both sides of (6.15) and using (6.16) on the resulting left-hand side, leads to the first-order ODE for $\Psi$,

$$
\Psi^{\prime} \Psi(u(t, \lambda))=(6-d) \Psi(u(t, \lambda))+u(t, \lambda)\left(u(t, \lambda)-\lambda_{d}\right), \quad \Psi(0)=0 .
$$

Letting $t \rightarrow 0$ and varying $\lambda$, we conclude that $\Psi$ is a solution of the ODE

$$
\Psi^{\prime} \Psi(u)=(6-d) \Psi(u)+u\left(u-\lambda_{d}\right), \quad u>0, \Psi(0)=0 .
$$

By using this equation to analyze the behavior of $\Psi$ near $\infty$, it is easy to see that in (6.14), $b=0$. The concavity of $\Psi$ implies that

$$
\lim _{u \downarrow 0} \frac{\Psi(u)}{u}=\lim _{u \downarrow 0} \Psi^{\prime}(u) \in(-\infty,+\infty] .
$$


If we divide both sides of (6.17) by $u$ and let $u \downarrow 0$, we conclude this limit, $\Psi^{\prime}(0)$ is in fact finite and satisfies

$$
\Psi^{\prime}(0)^{2}=(6-d) \Psi^{\prime}(0)-\lambda_{d},
$$

that is, $\Psi^{\prime}(0)=2$ if $d=2$, and $\Psi^{\prime}(0)=1$ or 2 if $d=3$. It is not hard to see using (6.8) that, in fact, $\Psi^{\prime}(0)=1$ if $d=3$. The fact that this derivative is finite, already implies that $\int_{0}^{\infty} \ell d \tilde{\pi}(\ell)<\infty$ and (6.14) can be rewritten as

$$
\Psi(u)=a_{d} u+\int_{0}^{\infty}\left(1-e^{-u \ell}-u \ell\right) d \pi(\ell), \quad \int_{0}^{\infty} \ell \wedge \ell^{2} d \pi(\ell)<\infty,
$$

where now $a_{d}=\Psi^{\prime}(0)=4-d$, by the above. The ODE (6.17) can be used to study the tail behaviour of $\Psi$, and hence $\pi$, via Tauberian theorems. For example, it is not hard to show that for some explicit $c_{6.19}>0$,

$$
\lim _{\varepsilon \downarrow 0} \varepsilon^{3 / 2} \pi([\varepsilon, \infty))=c_{6.19}
$$

The process of total mass of the exit measure from $B_{r}$ (as opposed to $G_{r_{0}-r}$ ) is studied in [6] as an inhomogeneous CSBP. The setting there is for general branching mechanisms, but the ideas used above and in defining $Z$ appear to be novel. It would be of interest to study the detailed behavior of the measure-valued process $r \rightarrow X_{G_{r_{0}-r}}$.

In [14], we instead worked with the exit measure from half-spaces $H_{r}=\left\{x: x_{1}<r\right\}$, where the total mass process is a $\Psi$-CSBP with $\Psi(u)=\frac{\sqrt{6}}{3} u^{3 / 2}$ (see [6] and Proposition 4.1 of [14]; it is interesting to note that the same $3 / 2$ stable branching mechanism appears in [13] for subordinate trees). The CSBP analysis in [14] was simpler due to this explicit 3/2-stable $\Psi$, but half-planes were clumsier and led to less precise results. See the discussion at the end of the Introduction. From the perspective of small jumps, the balls look like half-spaces and so (6.19) is to be expected. We hasten to add, however, that $\Psi(u)$ has behavior near $u=0$ (corresponding to $\pi$ at $\infty$ ) which is quite different and dimension dependent. Again using (6.17), one can show that $\forall \varepsilon>0$,

$$
\begin{aligned}
& u^{1+\varepsilon} \ll a_{d} u-\Psi(u) \ll u \quad \text { as } u \downarrow 0 \text { if } d=2, \\
& u^{2} \ll a_{d} u-\Psi(u) \ll u^{2-\varepsilon} \quad \text { as } u \downarrow 0 \text { if } d=3 .
\end{aligned}
$$

7. Proof of Propositions 1.6, 1.7. We use the notation from Section 6. In particular, $X_{0}$ and $r_{0}>0$ are as in $(6.1), Y_{r}(\cdot)=X_{G_{r_{0}-r}}(\cdot)$ for $0 \leq r<r_{0}$, and $Q_{X_{0}}(\cdot)=\mathbb{N}_{X_{0}}\left(\cdot \mid Y_{0}(1)>0\right)$.

In what follows, we always will work with the cadlag versions of $Y_{r}(1)$, and hence $Z_{t}$, constructed in Proposition 6.2(b) above. We let $W$ denote a generic snake under $\mathbb{N}_{X_{0}}$ or $Q_{X_{0}}$ with the associated "tip process" $\hat{W}(t)$ and excursion length $\sigma$. Define

$$
T_{0}(W)=\inf \left\{r \in\left[0, r_{0}\right): Y_{r}(1)=0\right\} \in\left[0, r_{0}\right] \quad \text { where } \inf \varnothing=r_{0}
$$

and

$$
\hat{T}_{0}(W)=\inf \{|\hat{W}(t)|: 0 \leq t \leq \sigma\}=\inf \{|x|: x \in \mathcal{R}\},
$$

the final equality holding $\mathbb{N}_{X_{0}}$-a.e. by (2.2). Clearly, we have

$$
Q_{X_{0}}(\cdot)=\mathbb{N}_{X_{0}}\left(\cdot \mid T_{0}>0\right) \text {. }
$$

LEMMA 7.1. The sets $\left\{T_{0}>0\right\}$ and $\left\{\hat{T}_{0}<r_{0}\right\}$ coincide $\mathbb{N}_{X_{0}}$-a.e., and on this set, $\hat{T}_{0}=$ $r_{0}-T_{0} \mathbb{N}_{X_{0}}$-a.e. 
PRoOF. For every rational $q$ in $\left[0, T_{0}\right), X_{G_{r_{0}-q}}(1)>0$ implies $\partial G_{r_{0}-q} \cap \mathcal{R}$ is nonempty (by (2.3)) and so by (2.2) $\hat{T}_{0} \leq r_{0}-q$. This proves that

$$
\hat{T}_{0} \leq r_{0}-T_{0} \quad \mathbb{N}_{X_{0}} \text {-a.e. on }\left\{T_{0}>0\right\} .
$$

Conversely, assume $r_{0}>T_{0}$ and choose rationals $q, q^{\prime}$ so that $T_{0}<q^{\prime}<q<r_{0}$. Then $X_{G_{r_{0}-q^{\prime}}}(1)=0$ and the special Markov property (Proposition 2.2(b)) at $R_{1}=r_{0}-q^{\prime}$ shows that $\mathbb{N}_{X_{0}}\left(\mathcal{R} \cap B_{r_{0}-q} \neq \varnothing \mid \mathcal{E}_{q^{\prime}}\right)=0$ a.e. on $\left\{T_{0}<q^{\prime}\right\}$. This proves that

$$
\hat{T}_{0} \geq r_{0}-T_{0} \quad \mathbb{N}_{X_{0}} \text {-a.e. on }\left\{T_{0}<r_{0}\right\} \text {. }
$$

The above is trivial if $T_{0}=r_{0}$ and so we have shown (by (7.2) and (7.3))

$$
\hat{T}_{0}=r_{0}-T_{0} \quad \mathbb{N}_{X_{0}} \text {-a.e. on }\left\{T_{0}>0\right\} \text {. }
$$

Finally, note that (7.2) shows $T_{0}>0$ implies $\hat{T}_{0}<r_{0}$, and (7.3) shows $T_{0}=0$ implies $\hat{T}_{0} \geq r_{0}$, which in turn shows $\hat{T}_{0}<r_{0}$ implies $T_{0}>0$ (all up to $\mathbb{N}_{X_{0}}$ null sets). This proves the a.e. equality of $\left\{T_{0}>0\right\}$ and $\left\{\hat{T}_{0}<r_{0}\right\}$, and completes the proof.

LEMMA 7.2. (a) For $0<r<r_{0}$,

$$
\begin{aligned}
\mathbb{N}_{X_{0}}\left(0<T_{0} \leq r\right)= & \mathbb{N}_{X_{0}}\left(r_{0}-r \leq \hat{T}_{0}<r_{0}\right) \\
= & \mathbb{N}_{X_{0}}\left(1\left(X_{G_{r_{0}}}(1)>0\right)\right. \\
& \left.\times \exp \left[-X_{G_{r_{0}}}(1)\left(r_{0}-r\right)^{2} U^{\infty, 1}\left(r_{0} /\left(r_{0}-r\right)\right)\right]\right) .
\end{aligned}
$$

(b) $\mathbb{N}_{X_{0}}\left(T_{0} \in d r\right) \ll d r$ on $\left\{0<r<r_{0}\right\}$ and $\mathbb{N}_{X_{0}}\left(\hat{T}_{0} \in d r\right) \ll d r$ on $\left\{0<r<2 r_{0}\right\}$.

PROOF. (a) Using (1.25) and scaling ((1.24) with $\lambda=\infty)$, we have for all $0<r<r_{0}$,

$$
\begin{aligned}
\mathbb{P}_{X_{G_{r_{0}}}}\left(X_{G_{r_{0}-r}}(1)=0\right) & =\exp \left(-X_{G_{r_{0}}}\left(U^{\infty, r_{0}-r}\right)\right) \\
& =\exp \left(-X_{G_{r_{0}}}(1)\left(r_{0}-r\right)^{-2} U^{\infty, 1}\left(r_{0} /\left(r_{0}-r\right)\right)\right) .
\end{aligned}
$$

The special Markov property (Proposition 2.2(a)(ii)) shows for $0<r<r_{0}$,

$$
\begin{aligned}
\mathbb{N}_{X_{0}}\left(0<T_{0} \leq r\right)= & \mathbb{N}_{X_{0}}\left(1\left(T_{0}>0\right) \mathbb{N}_{X_{0}}\left(X_{G_{r_{0}-r}}(1)=0 \mid \mathcal{E}_{0}\right)\right) \\
= & \mathbb{N}_{X_{0}}\left(1\left(T_{0}>0\right) \mathbb{P}_{X_{G_{r_{0}}}}\left(X_{G_{r_{0}-r}}(1)=0\right)\right) \\
= & \mathbb{N}_{X_{0}}\left(1\left(X_{G_{r_{0}}}(1)>0\right)\right. \\
& \left.\times \exp \left[-X_{G_{r_{0}}}(1)\left(r_{0}-r\right)^{-2} U^{\infty, 1}\left(r_{0} /\left(r_{0}-r\right)\right)\right]\right),
\end{aligned}
$$

where (7.4) has been used in the last line. This, together with Lemma 7.1, gives (a).

(b) The right-hand side of (a) is continuously differentiable in $r \in\left(0, r_{0}\right)$ because $U^{\infty, 1}$ is $C^{2}$ on $G_{1}$ (recall (1.27)). Here, we note that it is easy to justify differentiation inside the integral since $\mathbb{N}_{X_{0}}\left(X_{G_{r_{0}}}(1)>0\right)<\infty$ (recall (6.1)), $\mathbb{N}_{X_{0}}\left(X_{G_{r_{0}}}(1)\right)<\infty$ (recall (6.9)), and $\left(U^{\infty, 1}\right)^{\prime}(r)$ is bounded on compacts away from $\{r \leq 1\}$. This gives the first part of (b). Lemma 7.1 now implies the absolute continuity of $\mathbb{N}_{X_{0}}\left(\hat{T}_{0} \in d r\right)$ on $\left\{0<r<r_{0}\right\}$. But (6.1) allows us to replace $r_{0}$ with $\alpha r_{0}$ for any $1<\alpha<2$ in the above reasoning and so conclude that $\mathbb{N}_{X_{0}}\left(\hat{T}_{0} \in d r\right)$ is absolutely continuous on $\left\{0<r<2 r_{0}\right\}$.

Proof of Proposition 1.6 ASSUMing Proposition 1.7. By translation invariance, we may assume $x_{1}=0$. Fix $r_{0}, r_{1}$ and $X_{0}$ as in our hypotheses. We must show that

$$
X_{G_{r_{1}}}(1)=0 \quad \text { and } \quad X_{G_{r_{0}}}(1)>0 \quad \text { imply } \quad \operatorname{dim}\left(B_{r_{0}} \cap \partial \mathcal{R}\right) \geq d_{f} \quad \mathbb{P}_{X_{0}} \text {-a.s. }
$$


Measurability issues are easily handled using Lemma 2.1 and will henceforth be ignored. We work under $\mathbb{P}_{X_{0}}$ in the standard set-up and so from (2.4) have for $0<r \leq r_{0}$ and $J_{0}=\{j \in$ $\left.J: \hat{T}_{0}\left(W_{j}\right) \leq r_{0}\right\}$,

$$
\begin{aligned}
X_{G_{r}} & =\sum_{j \in J} X_{G_{r}}\left(W_{j}\right)=\sum_{j \in J} X_{G_{r}}\left(W_{j}\right) 1\left(\hat{T}_{0}\left(W_{j}\right) \leq r\right) \\
& =\sum_{j \in J_{0}} X_{G_{r}}\left(W_{j}\right) 1\left(\hat{T}_{0}\left(W_{j}\right) \leq r\right) .
\end{aligned}
$$

Here, we used the fact that $\hat{T}_{0}\left(W_{j}\right)>r$ implies $X_{G_{r}}\left(W_{j}\right)=0$ (e.g., by (2.3) and (2.2)). Recall from (2.2) that the range of the $j$ th excursion $W_{j}$ is

$$
\mathcal{R}_{j}:=\mathcal{R}\left(W_{j}\right)=\left\{\hat{W}_{j}(t): t \leq \sigma\left(W_{j}\right)\right\}
$$

It follows easily from (2.1) (see (2.19) in [14]) that for $x \in \overline{B_{r_{0}}}$,

$$
L^{x}=\sum_{j \in J_{0}} L^{x}\left(W_{j}\right)
$$

and, therefore,

$$
\begin{gathered}
\mathcal{R} \cap B_{r_{0}}=\bigcup_{j \in J_{0}}\left(\mathcal{R}_{j} \cap B_{r_{0}}\right) \quad \text { and so } \\
1_{\mathcal{R} \cap B_{r_{0}}}(x)=1\left(\sum_{j \in J_{0}} 1_{\mathcal{R}\left(W_{j}\right) \cap B_{r_{0}}}(x)>0\right) .
\end{gathered}
$$

We will frequently use the elementary topological result

$$
B_{r_{0}} \cap \partial F=B_{r_{0}} \cap \partial\left(B_{r_{0}} \cap F\right)=B_{r_{0}} \cap \partial\left(\overline{B_{r_{0}}} \cap F\right) \quad \text { for any closed set } F .
$$

It follows easily from Lemma 7.1 that

$$
T_{0}=r_{0}-\bigwedge_{j \in J_{0}} \hat{T}_{0}\left(W_{j}\right) \quad \text { on }\left\{T_{0}>0\right\}=\left\{\bigwedge_{j \in J_{0}} \hat{T}_{0}\left(W_{j}\right)<r_{0}\right\} \mathbb{P}_{X_{0}} \text {-a.s. }
$$

In view of the absolute continuity properties of $\hat{T}_{0}$ under $\mathbb{N}_{0}$ from Lemma 7.2 we see from the above that if $N_{0}=\left|J_{0}\right|$, a Poisson mean $\mathbb{N}_{X_{0}}\left(\hat{T}_{0} \leq r_{0}\right)$ random variable, then

$$
T_{0}>0 \quad \text { iff } \quad J_{0} \neq \varnothing \quad \text { iff } \quad N_{0}>0 \quad \mathbb{P}_{X_{0}} \text {-a.s. }
$$

By enlarging our probability space and randomizing the above Poisson points, we may assume that there is an i.i.d. sequence $\left\{\widetilde{W}_{j}: j \in \mathbb{N}\right\}$, independent of the Poisson variable $N_{0}=\left|J_{0}\right|$ with mean $\mathbb{N}_{X_{0}}\left(\hat{T}_{0} \leq r_{0}\right)$, and with common law

$$
\mathbb{N}_{X_{0}}\left(\cdot \mid \hat{T}_{0} \leq r_{0}\right)=\mathbb{N}_{X_{0}}\left(\cdot \mid \hat{T}_{0}<r_{0}\right)
$$

(the last equality by Lemma 7.2) and so that

$$
\sum_{j \in J_{0}} \delta_{W_{j}}=\sum_{j=1}^{N_{0}} \delta_{\widetilde{W}_{j}}
$$

Let $\widehat{W}_{j}$ denote the tip of the $j$ th excursion and define

$$
\begin{aligned}
& \hat{T}^{j}=\hat{T}_{0}\left(\widetilde{W}_{j}\right)<r_{0} \quad(\text { a.s. by }(7.11)), \\
& \tilde{\mathcal{R}}_{j}=\mathcal{R}\left(\widetilde{W}_{j}\right)=\left\{\widehat{W}_{j}(t): t \leq \sigma\left(\widetilde{W}_{j}\right)\right\} .
\end{aligned}
$$


Note that $X_{G_{r_{1}}}(1)=0$ implies $T_{0}<r_{0}-r_{1}$ a.s. and so, in view of (7.9),

$$
\begin{aligned}
& X_{G_{r_{1}}}(1)=0 \quad \text { and } \quad Y_{0}(1)=X_{G_{r_{0}}}(1)>0 \quad \text { imply } \quad \hat{T}^{j} \geq r_{1} \\
& \forall j \leq N_{0} \quad \mathbb{P}_{X_{0}} \text {-a.s. }
\end{aligned}
$$

The independence of the $\hat{T}^{j}$ 's and fact they have no positive atoms by Lemma 7.2 imply

$$
\mathbb{P}_{X_{0}}\left(\hat{T}^{j}=\hat{T}^{j^{\prime}} \text { for some } 1 \leq j \neq j^{\prime} \leq N_{0}, X_{G_{r_{1}}}(1)=0, X_{G_{r_{0}}}(1)>0\right)=0 \text {. }
$$

So on $\left\{X_{G_{r_{1}}}(1)=0, X_{G_{r_{0}}}(1)>0\right\}$ there is an a.s. unique $\tilde{j} \leq N_{0}$ such that $\hat{T}^{\tilde{j}}=\min \left\{\hat{T}^{j}\right.$ : $j \leq N_{0}$. . (7.14) and (7.13) imply that (if an empty minimum is $\left.r_{0}\right) \mathbb{P}_{X_{0}}$-a.s.,

$$
\begin{gathered}
\tilde{T}:=\min \left\{\hat{T}^{j}: j \neq \tilde{j}, j \leq N_{0}\right\}>\hat{T}^{\tilde{j}} \geq r_{1} \text { on } \\
\left\{X_{G_{r_{1}}}(1)=0, X_{G_{r_{0}}}(1)>0\right\} \subset\left\{N_{0} \geq 1\right\} .
\end{gathered}
$$

Hence $\tilde{T}$ is the largest radius $r \leq r_{0}$ so that a single excursion $\widetilde{W}_{j}$ enters $B_{r}$ (it exists on $\left.\left\{X_{G_{r_{1}}}(1)=0, X_{G_{r_{0}}}(1)>0\right\}\right)$.

By the definition of $\tilde{j}$ and $\tilde{T}$, we have from (7.15) and (7.7),

$$
B_{\tilde{T}} \cap \mathcal{R}=B_{\tilde{T}} \cap \tilde{\mathcal{R}}_{\tilde{j}} \quad \text { on }\left\{X_{G_{r_{1}}}(1)=0, X_{G_{r_{0}}}(1)>0\right\} \quad \text { a.s. }
$$

Therefore, using the above and (7.8) we obtain

$$
\begin{aligned}
\mathbb{P}_{X_{0}} & \left(\operatorname{dim}\left(B_{r_{0}} \cap \partial \mathcal{R}\right) \geq d_{f}, X_{G_{r_{1}}}(1)=0, X_{G_{r_{0}}}(1)>0\right) \\
& \geq \mathbb{P}_{X_{0}}\left(\operatorname{dim}\left(B_{\tilde{T}} \cap \partial \mathcal{R}\right) \geq d_{f}, X_{G_{r_{1}}}(1)=0, X_{G_{r_{0}}}(1)>0\right) \\
& =\mathbb{P}_{X_{0}}\left(\operatorname{dim}\left(B_{\tilde{T}} \cap \partial \tilde{\mathcal{R}}_{\tilde{j}}\right) \geq d_{f}, X_{G_{r_{1}}}(1)=0, X_{G_{r_{0}}}(1)>0\right) \\
& \geq \mathbb{P}_{X_{0}}\left(\left\{N_{0} \geq 1\right\} \cap\left(\bigcap_{j \leq N_{0}}\left\{\operatorname{dim}\left(B_{r} \cap \partial \tilde{\mathcal{R}}_{j}\right) \geq d_{f} \forall r>\hat{T}^{j} \geq r_{1}\right\}\right)\right) .
\end{aligned}
$$

In the last line we have used $N_{0} \geq 1$ iff $X_{G_{r_{0}}}(1)>0$ (by (7.10)), and on this set, $\hat{T}^{j} \geq r_{1}$ for all $j \leq N_{0}$ implies $T_{0} \leq r_{0}-r_{1}$ (by (7.9)) and so $X_{G_{r_{1}}}(1)=0$. We also use the fact (from (7.15)) that if $\left\{0<T_{0} \leq r_{0}-r_{1}\right\}$ then $\tilde{T}>\hat{T}^{\tilde{j}}$. The independence of the $\widetilde{W}_{j}$ 's and their joint independence from $N_{0}$ together with their common law in (7.11) imply that the right-hand side of (7.16) equals

$$
\mathbb{E}_{X_{0}}\left(1\left(N_{0} \geq 1\right) \prod_{j=1}^{N_{0}} \mathbb{N}_{X_{0}}\left(\operatorname{dim}\left(\partial \mathcal{R} \cap B_{r}\right) \geq d_{f} r>\hat{T}_{0} \geq r_{1} \mid \hat{T}_{0}<r_{0}\right)\right) .
$$

By Proposition 1.7 and Lemma 7.1, each of the terms in the above product equals $\mathbb{N}_{X_{0}}\left(\hat{T}_{0} \geq\right.$ $\left.r_{1} \mid \hat{T}_{0}<r_{0}\right)$ and so (7.17) equals

$$
\begin{aligned}
& \mathbb{E}_{X_{0}}\left(1\left(N_{0} \geq 1\right) 1\left(\bigwedge_{j=1}^{N_{0}} \hat{T}_{0}\left(\widetilde{W}_{j}\right) \geq r_{1}\right)\right) \\
& \quad=\mathbb{P}_{X_{0}}\left(0<T_{0} \leq r_{0}-r_{1}\right)=\mathbb{P}_{X_{0}}\left(X_{G_{r_{1}}}(1)=0, X_{G_{r_{0}}}(1)>0\right) .
\end{aligned}
$$

In the first equality we used (7.9) and (7.10). We have proved the left-hand side of (7.16) exceeds the above, and we conclude that

$$
X_{G_{r_{1}}}(1)=0 \quad \text { and } \quad X_{G_{r_{0}}}(1)>0 \quad \text { imply } \quad \operatorname{dim}\left(B_{r_{0}} \cap \partial \mathcal{R}\right) \geq d_{f} \quad \mathbb{P}_{X_{0}} \text {-a.s. }
$$

thus proving (7.5). 
Recall again that we always work with the cadlag version of $Y_{r}(1)$ from Proposition 6.2(b) which only has nonnegative jumps and is an $\left(\mathcal{E}_{r}^{+}\right)$supermartingale. Define a sequence of $\left(\mathcal{E}_{r}^{+}\right)$-stopping times by

$$
T_{n^{-1}}=\inf \left\{r \in\left[0, r_{0}\right): Y_{r}(1) \leq 1 / n\right\} \quad\left(\inf \varnothing=r_{0}\right) .
$$

Then

$$
\text { on }\left\{0<T_{0}\right\} \text { (and so } Q_{X_{0}} \text {-a.s.) } \quad T_{n^{-1}} \uparrow T_{0} \text { and } \quad T_{n^{-1}}<T_{0},
$$

where the last inequality holds since $Y_{r}(1)$ has no negative jumps. So under $Q_{X_{0}}, T_{0}$ is a predictable stopping time which is announced by $\left\{T_{n^{-1}}\right\}$ and so (see (12.9)(ii) in Chapter VI of [16])

$$
\mathcal{E}_{T_{0}-}^{+}=\bigvee_{n} \mathcal{E}_{T_{n}}^{+}
$$

Let $D_{r}=\left\{\operatorname{dim}\left(B_{r} \cap \partial \mathcal{R}\right) \geq d_{f}\right\}$ for $0<r \leq r_{0}$. We assume $\mathcal{E}_{r}^{+}$is augmented by $Q_{X_{0}}$-null sets throughout this section.

To complete the proof of Proposition 1.7, we need the following.

LEMMA 7.3. If $X_{0}=\delta_{x_{0}}$ where $\left|x_{0}\right| \geq 2 r_{0}$, then

$$
D_{r_{0}} \in \mathcal{E}_{T_{0}-}^{+} .
$$

For the proof of Proposition 1.7 below, it would suffice to show that $D_{r_{0}} \cap\left\{T_{0}<r_{0}\right\} \in \mathcal{E}_{T_{0}-}^{+}$, and this latter result should be intuitively obvious, as we now explain. With Lemma 7.1 in mind, we see that $\mathcal{E}_{T_{0}-}^{+}$includes information generated by the excursions of $W$ outside of its minimum radius. If this minimum radius is positive (as is the case on $\left\{T_{0}<r_{0}\right\}$ ), it is intuitively clear that this includes all the information generated by $W$. Even without intersecting with $\left\{T_{0}<r_{0}\right\}$, however, none of the mass that hits the origin will survive for any length of time and so again all of $W$ will have been observed. This last point stems from the fact that points are polar for Brownian motion in more than one dimension and be more formally justified using a mean measure result for the integral of the snake (Proposition 2 in Chapter IV of [12] with $p=1$ ).

PROOF OF LEMMA 7.3. Following the derivation of (7.11) in [14], one sees that in order to get (7.21), it is enough to show (cf. (7.18) of [14])

$$
\mathbb{N}_{x_{0}}\left(\int_{0}^{\infty} 1\left(\inf _{v \leq \zeta_{u}}\left|W_{u}(v)\right|=\hat{T}_{0}\right) d u\right)=0 .
$$

Next, follow the proof of (7.18) in [14] using the historical process and its Palm measure formula, to bound the left-hand side of (7.22) by (cf. (7.22) of [14])

$$
\int_{0}^{\infty} E_{x_{0}}^{B}\left(\exp \left(-\int_{0}^{s} \frac{2(4-d)}{\left|B_{t}-m_{s}\right|^{2}} d t\right)\right) d s .
$$

Here, $B$ denotes a $d$-dimensional Brownian motion starting at $x_{0}$ under $P_{x_{0}}^{B}$ and $m_{s}=$ $\inf _{s^{\prime} \leq s}\left|B_{s^{\prime}}\right|$. A simple application of Lévy's modulus for $B$ shows that $\int_{0}^{s} \frac{2(4-d)}{\left|B_{t}-m_{s}\right|^{2}} d t$ is infinite a.s. and so proves (7.22) as required. More details may be found in Supplementary Material [9] where the actual definition of $\mathcal{E}_{r}$ is even used.

Proof of Proposition 1.7. Clearly, it suffices to fix $x_{0} \in \operatorname{Supp}\left(X_{0}\right)$ and prove the result with $\mathbb{N}_{x_{0}}$ in place of $\mathbb{N}_{X_{0}}$. By translation invariance, we may assume $x_{1}=0$, and so 
$\left|x_{0}\right| \geq 2 r_{0}$. Fix $0<r_{1}<r_{0}$. Assume $0 \leq r<r_{0}$ and $n \in \mathbb{N}$ is large enough so that $r+n^{-1}<$ $r_{0}$. By Lemma 2.1(b), there is a universally measurable map $\psi: \mathcal{K} \rightarrow[0,1]$ such that

$$
\begin{aligned}
1\left(D_{r_{0}-r-n^{-1}}\right) & \left.=1\left(\operatorname{dim}\left(\partial\left(\bar{B}_{r_{0}-r-n^{-1}} \cap \mathcal{R}\right) \cap B_{r_{0}-r-n^{-1}}\right) \geq d_{f}\right) \quad \text { (by (7.8) }\right) \\
& =\psi\left(\bar{B}_{r_{0}-r-n^{-1}} \cap \mathcal{R}\right) .
\end{aligned}
$$

Recall that conditional expectations with respect to $\mathcal{E}_{r}$, under $\mathbb{N}_{x_{0}}$ and $Q_{x_{0}}$, agree $Q_{x_{0}}$-a.s., and note that Proposition 2.2(b) can be trivially extended to universally measurable maps. Therefore, up to $Q_{x_{0}}$-null sets, on the event $\left\{4 n^{-2} \leq Y_{r}(1) \leq\left(r_{0}-r\right)^{2}\right\}\left(\in \mathcal{E}_{r}\right)$ we have

$$
\begin{aligned}
Q_{x_{0}}\left(D_{r_{0}} \mid \mathcal{E}_{r}\right) & \geq Q_{x_{0}}\left(D_{r_{0}-r-n^{-1}} \mid \mathcal{E}_{r}\right) \\
& =\mathbb{P}_{Y_{r}}\left(D_{r_{0}-r-n^{-1}}\right) \quad(\text { by }(7.23) \text { and Proposition 2.2(b) }) \\
& \geq \mathbb{P}_{Y_{r}}\left(\operatorname{dim}\left(\partial \mathcal{R} \cap B_{r_{0}-r-\left(\sqrt{Y_{r}(1)} / 2\right)}\right) \geq d_{f}\right) \\
& \geq q_{5.4},
\end{aligned}
$$

where Lemma 5.4 and the assumed bounds on $Y_{r}(1)$ are used in the last inequality, and the assumed lower bound on $Y_{r}(1)$ is used in the next to last inequality. Let $n \rightarrow \infty$ and take limits from above in $r \in \mathbb{Q}_{+}$(recall $Y_{r}(1)$ is cadlag) to conclude that

$$
\begin{aligned}
M_{r} & :=Q_{x_{0}}\left(D_{r_{0}} \mid \mathcal{E}_{r}^{+}\right) \geq q_{5.4} \text { on }\left\{0<Y_{r}(1)<\left(r_{0}-r\right)^{2}\right\} \\
\forall r & \in \mathbb{Q} \cap\left(0, r_{0}\right) \quad Q_{x_{0}} \text {-a.s. }
\end{aligned}
$$

Here, $M_{r}$ is a cadlag version of the bounded martingale on the left-hand side. Using rightcontinuity, one can strengthen (7.24) to

$$
\begin{aligned}
& M_{r}=Q_{x_{0}}\left(D_{r_{0}} \mid \mathcal{E}_{r}^{+}\right) \geq 95.4 \quad \text { on }\left\{0<Y_{r}(1)<\left(r_{0}-r\right)^{2}\right\} \\
& \quad \forall r \in\left(0, r_{0}\right) \quad Q_{x_{0}} \text {-a.s. }
\end{aligned}
$$

On $\left\{0<T_{0} \leq r_{0}-r_{1}\right\}$, we have from (7.19) and the lack of negative jumps for $Y_{r}(1)$, for $n$ large,

$$
T_{n^{-1}} \in\left(0, r_{0}-r_{1}\right) \quad \text { and } \quad Y_{T_{n^{-1}}}(1)=n^{-1}<\left(r_{0}-T_{1 / n}\right)^{2} \quad Q_{x_{0}} \text {-a.s. }
$$

By Corollary (17.10) in Chapter VI of [16], (7.25) and (7.26), we have $Q_{x_{0}}$-a.s. on $\left\{0<T_{0} \leq\right.$ $\left.r_{0}-r_{1}\right\} \in \mathcal{E}_{T_{0}-}^{+}$,

$$
Q_{x_{0}}\left(D_{r_{0}} \mid \mathcal{E}_{T_{0}-}^{+}\right)=\lim _{n \rightarrow \infty} M\left(T_{n^{-1}}\right) \geq q_{5.4}
$$

Multiplying the above by $1\left(\left\{0<T_{0} \leq r_{0}-r_{1}\right\}\right)$, we see from Lemma 7.3 that

$$
1\left(D_{r_{0}} \cap\left\{0<T_{0} \leq r_{0}-r_{1}\right\}\right) \geq q_{5.4} 1\left(\left\{0<T_{0} \leq r_{0}-r_{1}\right\}\right) \quad Q_{x_{0}} \text {-a.s. }
$$

and, therefore, by Lemma 7.1,

$$
r_{1} \leq \hat{T}_{0}<r_{0} \quad \text { implies } \quad \operatorname{dim}\left(B_{r_{0}} \cap \partial \mathcal{R}\right) \geq d_{f} \quad Q_{x_{0}} \text {-a.s. }
$$

This remains true if we replace $r_{0}$ by any $r \in\left(r_{1}, r_{0}\right]$ since $B_{2 r} \subset \operatorname{Supp}\left(X_{0}\right)^{c}$ still holds. Therefore, we may fix $\omega$ outside a $Q_{x_{0}}$-null set so that for any rational $r \in \mathbb{Q}$ such that $r \in\left(r_{1}, r_{0}\right], r_{1} \leq \hat{T}_{0}<r$ implies $\operatorname{dim}\left(B_{r} \cap \partial \mathcal{R}\right) \geq d_{f}$. By monotonicity of the conclusion in $r$, this means that $\left\{r_{1} \leq \hat{T}_{0}<r_{0}\right\}$ implies $\operatorname{dim}\left(B_{r} \cap \partial \mathcal{R}\right) \geq d_{f}$ for all $r>\hat{T}_{0}$. This gives Proposition 1.7 under $Q_{x_{0}}$. The result under $\mathbb{N}_{x_{0}}$ is now immediate from the definition of $Q_{x_{0}}$, and $\left\{Y_{0}(1)>0\right\}=\left\{\hat{T}_{0}<r_{0}\right\} \mathbb{N}_{x_{0}}$-a.e. (by Lemma 7.1). 


\section{APPENDIX}

A.1. Proof of Lemma 2.1. (a) Let $K^{(\varepsilon)}=\{x: d(x, K)<\varepsilon\}$. If $K_{0} \in \mathcal{K}$ and $0<r \leq 1$ are fixed, it suffices to show that $\left\{K \in \mathcal{K}: \rho\left(K \cap \bar{B}_{R}, K_{0}\right)<r\right\}$ is Borel. If $r_{n} \uparrow r$, this set is equal to

$$
\begin{aligned}
\{K & \left.: K \cap \bar{B}_{R} \subset K_{0}^{(r)}\right\} \cap\left\{K: K_{0} \subset\left(K \cap \bar{B}_{R}\right)^{(r)}\right\} \\
& =\left\{K: K \cap \bar{B}_{R} \subset K_{0}^{(r)}\right\} \cap\left(\bigcup_{n=1}^{\infty}\left\{K: K_{0} \subset\left(K \cap \bar{B}_{R}\right)^{r_{n}}\right\}\right) \\
& :=S_{1} \cap\left(\bigcup_{n=1}^{\infty} S_{2}^{n}\right) .
\end{aligned}
$$

It is then not hard to show that $S_{1}$ is open in $\mathcal{K}$ and $S_{2}^{n}$ is closed in $\mathcal{K}$.

(b) This easily reduces to showing that for any fixed rationals $q \in(0, \alpha)$ and $r \in(0, R)$, the following describes an universally measurable subset of $K^{\prime} s$ in $\mathcal{K}$ :

For any natural number $N$, there is a finite number of open balls

$$
\begin{aligned}
& B^{1}, \ldots, B^{M} \text { centered at points in } \mathbb{Q}^{d} \text { and with rational radii } \\
& r_{1}, \ldots, r_{M}>0 \text { satisfying } \sum_{i=1}^{M} r_{i}^{q}<N^{-1} \text { so that } \partial K \cap \bar{B}_{r} \subset \bigcup_{i=1}^{M} B^{i} .
\end{aligned}
$$

So fixing $B^{i}$ and $r$ as above, it suffices to show

$$
A_{1}=\left\{K \in \mathcal{K}: \partial K \cap \bar{B}_{r} \subset \bigcup_{i=1}^{M} B^{i}\right\}^{c} \text { is an analytic set in } \mathcal{K},
$$

because this implies $A_{1}$, and hence $A_{1}^{c}$, is a universally measurable set in $\mathcal{K}$. Let $K_{0}=$ $\left(\bigcup_{i=1}^{M} B^{i}\right)^{c} \cap \bar{B}_{r} \in \mathcal{K}, H_{0}=\left\{(x, K) \in \mathbb{R}^{d} \times \mathcal{K}: x \in K\right\}$, and for $n \geq 1$, set $H_{n}=\{(x, K) \in$ $\left.\mathbb{R}^{d} \times \mathcal{K}: B_{n^{-1}}(x) \not \subset K\right\}$. Then

$$
\begin{aligned}
A_{1} & =\left\{K \in \mathcal{K}: K_{0} \cap \partial K \neq \varnothing\right\} \\
& =\left\{K \in \mathcal{K}: \exists x \in K_{0} \text { s.t. } x \in K \text { and } \forall n \in \mathbb{N} B_{n^{-1}}(x) \cap K^{c} \neq \varnothing\right\} \\
& =\left\{K \in \mathcal{K}: \exists x \in \mathbb{R}^{d} \text { s.t. }(x, K) \in\left(K_{0} \times \mathcal{K}\right) \cap\left(\bigcap_{n=0}^{\infty} H_{n}\right)\right\} .
\end{aligned}
$$

Using the well-known fact that the projection of a Borel subset of $K_{0} \times \mathcal{K}$ onto $\mathcal{K}$ is an analytic subset of $\mathcal{K}$ (see, e.g., Theorem 13 in Chapter III of [5] and note the argument goes through with $\mathbb{R}^{d}$ in place of $\mathbb{R}$ ), it then suffices to show each $H_{n}$ is Borel. First one can check that $H_{0}$ is the countable intersection of the open sets $H_{0}^{M}=\left\{(x, K): x \in K^{(1 / M)}\right\}$. Next, it is not hard to see that $H_{n}$ is open for $n \geq 1$, and we are done.

A.2. Proof of Lemma 4.5. For the proof, we will use the following lemma of Marc Yor (see Proposition 2.5 of [14] and [20]). Recall that for $\gamma \in \mathbb{R},\left(\rho_{t}\right)$ denotes a $\gamma$-dimensional Bessel process starting from $r>0$ under $P_{r}^{(\gamma)},\left(\mathcal{F}_{t}\right)$ is the filtration generated by $\rho$ and $\tau_{R}=\inf \left\{t \geq 0: \rho_{t} \leq R\right\}$.

LEMMA A.1. Let $\lambda \geq 0, \mu \in \mathbb{R}, r>0$ and $v=\sqrt{\lambda^{2}+\mu^{2}}$. If $\Phi_{t} \geq 0$ is $\mathcal{F}_{t}$-adapted, then for all $R<r$, we have

$$
E_{r}^{(2+2 \mu)}\left(\Phi_{t \wedge \tau_{R}} \exp \left(-\frac{\lambda^{2}}{2} \int_{0}^{t \wedge \tau_{R}} \frac{1}{\rho_{s}^{2}} d s\right)\right)=r^{\nu-\mu} E_{r}^{(2+2 v)}\left(\left(\rho_{t \wedge \tau_{R}}\right)^{-\nu+\mu} \Phi_{t \wedge \tau_{R}}\right) .
$$


Now we are ready to give the following.

Proof of Lemma 4.5. We use Fatou's lemma and then Lemma A.1 to get that for $a \geq 0$,

$$
\begin{aligned}
& E_{x}\left(1_{\left(\tau_{R}<\infty\right)} \exp \left(\int_{0}^{\tau_{R}} \frac{a}{\left|B_{S}\right|^{q}} d s\right) \exp \left(-\int_{0}^{\tau_{R}} \frac{2(4-d)-\zeta / 2}{\left|B_{s}\right|^{2}} d s\right)\right) \\
& \leq \liminf _{t \rightarrow \infty} E_{|x|}^{(2+2 \mu)}\left(1_{\left(\tau_{R} \leq \tau_{R} \wedge t\right)} \exp \left(\int_{0}^{\tau_{R} \wedge t} \frac{a}{\rho_{s}^{q}} d s\right)\right. \\
&\left.\quad \times \exp \left(-\int_{0}^{\tau_{R} \wedge t} \frac{2(4-d)-\zeta / 2}{\rho_{s}^{2}} d s\right)\right) \\
&=|x|^{v_{\zeta}-\mu} \liminf _{t \rightarrow \infty} E_{|x|}^{\left(2+2 v_{\zeta}\right)}\left(1_{\left(\tau_{R} \leq \tau_{R} \wedge t\right)} \exp \left(\int_{0}^{\tau_{R} \wedge t} \frac{a}{\rho_{s}^{q}} d s\right) \rho_{t \wedge \tau_{R}}^{\mu-v_{\zeta}}\right) \\
&=(R /|x|)^{\mu-v_{\zeta}} E_{|x|}^{\left(2+2 v_{\zeta}\right)}\left(1_{\left(\tau_{R}<\infty\right)} \exp \left(\int_{0}^{\tau_{R}} \frac{a}{\rho_{s}^{q}} d s\right)\right) \\
&\left(\text { since } \rho_{t \wedge \tau_{R}}=R \text { on }\left\{\tau_{R} \leq t\right\}\right) \\
&=(R /|x|)^{p_{\zeta}} E_{|x|}^{\left(2+2 v_{\zeta}\right)}\left(\exp \left(\int_{0}^{\tau_{R}} \frac{a}{\rho_{s}^{q}} d s\right) \mid \tau_{R}<\infty\right),
\end{aligned}
$$

where in next to the last line we use monotone convergence for $a \geq 0$, and in the last line the hitting probabilities for Bessel processes (e.g., (48.3) and (48.5) in Chapter V of [16]) as well as $p_{\zeta}=\mu+v_{\zeta}$. Note that for $a<0$, by bounded convergence, we get equality in the second line above (with $\liminf _{t \rightarrow \infty}$ replaced by $\lim _{t \rightarrow \infty}$ ), and thus proceeding as above we get, by using bounded convergence again in the next to the last line, that (4.10) holds for $a<0$.

It remains to verify the lower bound in (4.10), for $a \geq 0$. Fix $T>0$. Then we have

$$
\begin{aligned}
& E_{x}\left(1\left(\tau_{R}<\infty\right) \exp \left(\int_{0}^{\tau_{R}} \frac{a}{\left|B_{S}\right|^{q}} d s\right) \exp \left(-\int_{0}^{\tau_{R}} \frac{2(4-d)-\zeta / 2}{\left|B_{S}\right|^{2}} d s\right)\right) \\
& \quad \geq E_{|x|}^{(2+2 \mu)}\left(1_{\left(\tau_{R}<\infty\right)} \exp \left(\int_{0}^{\tau_{R} \wedge T} \frac{a}{\rho_{s}^{q}} d s\right) \exp \left(-\int_{0}^{\tau_{R}} \frac{2(4-d)-(\zeta / 2)}{\rho_{s}^{2}} d s\right)\right) \\
& \quad=|x|^{\nu_{\zeta}-\mu} \lim _{t \rightarrow \infty} E_{|x|}^{\left(2+2 v_{\zeta}\right)}\left(1_{\left(\tau_{R} \leq \tau_{R} \wedge t\right)} \exp \left(\int_{0}^{\tau_{R} \wedge t \wedge T} \frac{a}{\rho_{S}^{q}} d s\right) R^{\mu-v_{\zeta}}\right) \\
& =(R /|x|)^{\mu-v_{\zeta}} E_{|x|}^{\left(2+2 v_{\zeta}\right)}\left(1_{\left(\tau_{R}<\infty\right)} \exp \left(\int_{0}^{\tau_{R} \wedge T} \frac{a}{\rho_{s}^{q}} d s\right)\right) \\
& =(R /|x|)^{p_{\zeta}} E_{|x|}^{\left(2+2 v_{\zeta}\right)}\left(\exp \left(\int_{0}^{\tau_{R} \wedge T} \frac{a}{\rho_{s}^{q}} d s\right) \mid \tau_{R}<\infty\right),
\end{aligned}
$$

where in the first equality we used bounded convergence and Lemma A.1, in the second equality bounded convergence again, and in the last equality the hitting probabilities for Bessel processes. Now let $T \rightarrow \infty$, to get the required lower bound, and we are done.

Acknowledgements. We thank an anonymous referee for making several suggestions which have improved the presentation of our paper.

The first and third authors were supported by an NSERC Discovery Grant.

The second author was supported by the Israel Science Foundation Grants 1325/14 and $1704 / 18$. 


\section{SUPPLEMENTARY MATERIAL}

Supplement to "On the topological boundary of the range of super-Brownian motion" (DOI: 10.1214/19-AOP1386SUPP; .pdf). The supplementary material contains the proof of Proposition 5.1 and gives more details of the proof of Lemma 7.3.

\section{REFERENCES}

[1] Abraham, R. and Le Gall, J.-F. (1994). Sur la mesure de sortie du super mouvement brownien. Probab. Theory Related Fields 99 251-275. MR1278885 https://doi.org/10.1007/BF01199025

[2] Brezis, H., Peletier, L. A. and Terman, D. (1986). A very singular solution of the heat equation with absorption. Arch. Ration. Mech. Anal. 95 185-209. MR0853963 https://doi.org/10.1007/BF00251357

[3] Dawson, D. A., Iscoe, I. and Perkins, E. A. (1989). Super-Brownian motion: Path properties and hitting probabilities. Probab. Theory Related Fields 83 135-205. MR1012498 https://doi.org/10.1007/ BF00333147

[4] Dawson, D. A. and Perkins, E. A. (1991). Historical processes. Mem. Amer. Math. Soc. 93 iv+179. MR1079034 https://doi.org/10.1090/memo/0454

[5] Dellacherie, C. and Meyer, P.-A. (1978). Probabilities and Potential. North-Holland Mathematics Studies 29. North-Holland, Amsterdam. MR0521810

[6] Hesse, M. and Kyprianou, A. E. (2014). The mass of super-Brownian motion upon exiting balls and Sheu's compact support condition. Stochastic Process. Appl. 124 2003-2022. MR3188347 https://doi.org/10.1016/j.spa.2014.01.011

[7] Hong, J. (2018). Renormalization of local times of super-Brownian motion. Electron. J. Probab. 23 Article ID 109. MR3878134 https://doi.org/10.1214/18-ejp231

[8] Hong, J. (2019). Improved Hölder continuity near the boundary of one-dimensional super-Brownian motion. Electron. Commun. Probab. 24 Article ID 28. MR3962478 https://doi.org/10.1214/19-ECP237

[9] Hong, J. Mytnik, L. and Perkins, E. (2020). Supplement to "On the topological boundary of the range of super-Brownian motion." https://doi.org/10.1214/19-AOP1386SUPP.

[10] IsCOE, I. (1988). On the supports of measure-valued critical branching Brownian motion. Ann. Probab. 16 200-221. MR0920265

[11] LE Gall, J.-F. (1995). The Brownian snake and solutions of $\Delta u=u^{2}$ in a domain. Probab. Theory Related Fields 102 393-432. MR1339740 https://doi.org/10.1007/BF01192468

[12] Le Gall, J.-F. (1999). Spatial Branching Processes, Random Snakes and Partial Differential Equations. Lectures in Mathematics ETH Zürich. Birkhäuser, Basel. MR1714707 https://doi.org/10.1007/ 978-3-0348-8683-3

[13] Le Gall, J.-F. (2018). Subordination of trees and the Brownian map. Probab. Theory Related Fields 171 819-864. MR3827223 https://doi.org/10.1007/s00440-017-0794-9

[14] MYtnik, L. and Perkins, E. (2019). The dimension of the boundary of super-Brownian motion. Probab. Theory Related Fields 174 821-885. MR3980306 https://doi.org/10.1007/s00440-018-0866-5

[15] Perkins, E. (2002). Dawson-Watanabe superprocesses and measure-valued diffusions. In Lectures on Probability Theory and Statistics (Saint-Flour, 1999). Lecture Notes in Math. 1781 125-324. Springer, Berlin. MR1915445

[16] Rogers, L. C. G. and Williams, D. (2000). Diffusions, Markov Processes, and Martingales, Vol. 2: Itô Calculus. Cambridge Mathematical Library. Cambridge Univ. Press, Cambridge. MR1780932 https://doi.org/10.1017/CBO9781107590120

[17] Silverstein, M. L. (1967/1968). A new approach to local times. J. Math. Mech. 17 1023-1054. MR0226734

[18] Sugitani, S. (1989). Some properties for the measure-valued branching diffusion processes. J. Math. Soc. Japan 41 437-462. MR0999507 https://doi.org/10.2969/jmsj/04130437

[19] TAYLOR, S. J. (1961). On the connexion between Hausdorff measures and generalized capacity. Proc. Camb. Philos. Soc. 57 524-531. MR0133420 https://doi.org/10.1017/s0305004100035581

[20] Yor, M. (1992). On some exponential functionals of Brownian motion. Adv. in Appl. Probab. 24 509-531. MR1174378 https://doi.org/10.2307/1427477 